\title{
Phase transitions in a three-dimensional analogue of Klebanov-Strassler
}

\author{
Daniel Elander, ${ }^{a}$ Antón F. Faedo, ${ }^{b, c, d}$ David Mateos $^{b, e}$ and Javier G. Subils ${ }^{b}$ \\ ${ }^{a}$ Laboratoire Charles Coulomb (L2C), University of Montpellier, CNRS, \\ Montpellier, France \\ ${ }^{b}$ Departament de Física Quàntica i Astrofísica 8 Institut de Ciències del Cosmos (ICC), \\ Universitat de Barcelona, \\ Martí Franquès 1, ES-08028, Barcelona, Spain \\ ${ }^{c}$ Departamento de Física, Universidad de Oviedo, \\ Federico García Lorca 18, ES-33007, Oviedo, Spain \\ ${ }^{d}$ Instituto Universitario de Ciencias y Tecnologías Espaciales de Asturias (ICTEA), \\ Calle de la Independencia 13, ES-33004, Oviedo, Spain \\ ${ }^{e}$ Institució Catalana de Recerca i Estudis Avançats (ICREA), \\ Passeig Lluís Companys 23, ES-08010, Barcelona, Spain \\ E-mail: daniel.elander@umontpellier.fr, anton.faedo@uniovi.es, \\ dmateos@fqa.ub.edu, jgsubils@fqa.ub.edu
}

ABSTRACT: We use top-down holography to study the thermodynamics of a one-parameter family of three-dimensional, strongly coupled Yang-Mills-Chern-Simons theories with Mtheory duals. For generic values of the parameter, the theories exhibit a mass gap but no confinement, meaning no linear quark-antiquark potential. For two specific values of the parameter they flow to an infrared fixed point or to a confining vacuum, respectively. As in the Klebanov-Strassler solution, on the gravity side the mass gap is generated by the smooth collapse to zero size of a cycle in the internal geometry. We uncover a rich phase diagram with thermal phase transitions of first and second order, a triple point and a critical point.

Keywords: Black Holes in String Theory, Confinement, Gauge-gravity correspondence

ARXIV EPRINT: 2002.08279 


\section{Contents}

1 Introduction 1

2 Low-temperature phases $\quad 3$

3 High-temperature phases $\quad 6$

3.1 Boundary conditions

3.2 Numerical integration 8

$\begin{array}{lll}3.3 & \text { Zero-entropy limit } & 10\end{array}$

4 Thermodynamics and the phase diagram 14

4.1 Thermodynamic quantities 14

$\begin{array}{lll}4.2 & \text { Phase diagram } & 15\end{array}$

4.3 Quasi-conformal thermodynamics 20

4.4 Quasi-confining thermodynamics 20

$\begin{array}{ll}4.5 & \text { Regime of validity } \\ \end{array}$

5 Conclusions and discussion $\quad 22$

A Ansatz and equations of motion $\quad 26$

A.1 Ten-dimensional ansatz 26

A.2 Four-dimensional reduction 28

B UV expansions $\quad 30$

C Expansions near the horizon $\quad 33$

D Numerical output $\quad 34$

\section{Introduction}

Three-dimensional gauge theories enjoy properties that can challenge our four-dimensional intuition. A prominent example is the existence of the Chern-Simons (CS) term, which is a relevant deformation of the Yang-Mills action. Despite being topological, it has dramatic effects on the dynamics. Besides, since the gauge coupling has positive mass dimension, three-dimensional Yang-Mills theories admit a free ultraviolet (UV) fixed point. Conversely, they are generically strongly coupled in the infrared (IR). This gives rise to interesting nonperturbative phenomena, including the appearance of novel quantum phases, which may play a role in condensed matter systems where the gauge symmetry is typically emergent. These new phases cannot be uncovered using semiclassical approximations such as the 
large-CS level, large-number of flavours or large-mass limits, but their existence can be inferred employing dualities, anomaly matching or other arguments, as in [1-4]. As usual, supersymmetry is a valuable tool in this type of analyses [5], and lattice techniques can also be adapted to these kinds of models [6-8].

Holography also provides a useful tool to study strongly coupled gauge theories, especially when the approaches above are not applicable. In this paper we will use this tool to study a one-parameter family of three-dimensional gauge theories at non-zero temperature by means of their gravitational duals. The interactions consist of Yang-Mills interactions associated to a two-sites quiver, similarly to the Klebanov-Witten theory [9], as well as CS terms for both gauge groups. The parameter distinguishing one theory from another, which we call $b_{0}$, is related to the difference between the inverse squared couplings of each of the two gauge groups. In our conventions $b_{0}$ takes values in the interval $[0,1]$. The gravitational duals at zero temperature can be described in ten- or eleven-dimensional supergravity and are therefore firmly embedded in string or M-theory. They preserve $\mathcal{N}=1$ supersymmetry and were studied in detail in [10], based on the results of $[11,12]$. For $0<b_{0}<1$ the theories exhibit a mass gap but no confinement in the sense of a linear quark-antiquark potential at large distances [10]. For $b_{0}=0$ there is no mass gap and the theory flows to a fixed point in the IR. For $b_{0}=1$ it exhibits not just a mass gap but also confinement. These properties are pictorially represented in figure 1.

The aim of this work is to study the thermodynamics of the family of theories above. The results are summarised in figure 10. Generically, as the temperature increases gradually from zero, the system undergoes a phase transition from the gapped state to an ungapped phase. The ungapped nature of this phase follows from the fact that, on the gravity side, it is characterized by the presence of a black brane horizon, which supports excitations with arbitrarily low energy such as hydrodynamic modes. Therefore we will refer to this phase transition as a "degapping transition." Its details and the behaviour of the system at even higher temperatures depend on the value of $b_{0}$ as follows.

For $b_{0} \in\left(b_{0}^{\text {triple }}, 1\right]$, with $b_{0}^{\text {triple }} \approx 0.6847$, there is a first-order, Hawking-Page-like phase transition between the gapped geometry and the black brane geometry. As expected, the entropy density jumps discontinuously from zero to a positive value across this phase transition. The critical temperature depends on $b_{0}$, as indicated by the dashed, red curve in figure 10. In the particular case $b_{0}=1$ the phase transition is a deconfinement phase transition. For these values of $b_{0}$ no other phase transitions occur as the temperature is further increased.

For $b_{0} \in\left(0, b_{0}^{\text {triple }}\right)$, the system also undergoes a phase transition from the gapped to the black brane geometry, as indicated by the solid, magenta curve in figure 10. However, in this case the entropy density increases continuously from zero. In other words, from the viewpoint of the entropy density alone the degapping transition for $b_{0} \in\left(0, b_{0}^{\text {triple }}\right)$ looks like a second-order phase transition. In spite of this, we expect other quantities to change discontinuously, and hence the transition to be still first-order. The reason is that the geometry changes discontinuously. The continuity of the entropy is simply due to the fact that this discontinuity takes place between two solutions with zero horizon area. Therefore we expect that, although their calculation is beyond the scope of this 
paper, other quantities such as $n$-point functions, which probe the geometry at different scales, will change discontinuously. For $b_{0} \in\left(0, b_{0}^{\text {critical }}\right)$, with $b_{0}^{\text {critical }} \approx 0.6815<b_{0}^{\text {triple }}$, no other phase transitions take place as the temperature is further increased. In contrast, for $b_{0} \in\left(b_{0}^{\text {critical }}, b_{0}^{\text {triple }}\right)$ a second phase transition occurs as the temperature is further increased, as indicated by the dashed, black line in figure 10. In this case this is a first-order transition between two black-brane solutions with a discontinuous jump in the entropy density. By continuity, it follows that this line of phase transitions ends at a critical point at $b_{0}=b_{0}^{\text {critical }}$, at which the phase transition is second-order. The three curves of phase transitions in figure 10 meet at a triple point at $b_{0}=b_{0}^{\text {triple }}$, at which the three phases can coexist. For $b_{0}=0$ there are no phase transitions at any temperature.

Some properties of our system are similar to those of the Klebanov-Strassler (KS) solution [13], which is dual to a four-dimensional gauge theory. For example, the mass gap at zero temperature arises from the shrinking to zero size of a cycle in the internal part of the geometry. This means that our theories are truly three-dimensional at all energy scales, as opposed to Witten-like models [14] in which the gap arises from a compact gauge theory direction that becomes important at high energies. A further similarity with the KS case $[15,16]$ is the presence of a phase transition from a gapped to an ungapped phase. There are two main differences between our system and the KS model. The first one is that our theories have a simple, weakly coupled UV completion, since they are asymptotically free. The second one is that these theories come in a one-parameter family, which gives rise to a rich phase diagram including a critical point and a triple point.

The organization of the paper is as follows. First, in section 2 we discuss the lowtemperature phases. Since these are constructed directly from the zero-temperature solutions, we review their properties and give the details of the expected gauge theory duals. In section 3 we construct numerically the competing phases, which take the form of black brane solutions. Equipped with all these solutions, we explore their thermodynamic properties and present the relevant phase diagram in section 4 . We finally discuss the results and conclude in section 5. Most technical aspects are relegated to several appendices.

\section{Low-temperature phases}

The ground state of the system corresponds to the regular supersymmetric solutions of [10]. These take the form of a stack of $N$ coincident M2-branes where the transverse space is one of the eight-dimensional manifolds pertaining to the $\mathbb{B}_{8}$ class, found originally in [11, 12]. These metrics have $\operatorname{Spin}(7)$ holonomy in order to preserve $\mathcal{N}=1$ supersymmetry. They come in a one-parameter family, characterised for instance by the value of the radial coordinate at which the geometry ends smoothly. Moreover, they posses a non-trivial four-cycle that does not collapse at the end-of-space and whose size provides a scale and consequently a mass gap in the dual theory. Nevertheless, there is no confinement in the sense explained in [10].

Regularity of the solution is ensured by the presence of a complicated four-form, with different internal components. We should mention that, once we pick one of the $\mathbb{B}_{8}$ metrics, 
specified by the scale of the mass gap or, equivalently, by the position of the end-of-space, the four-form is completely fixed by the requirement that the entire geometry be regular.

The UV of the model is best understood by reducing on the appropriate internal circle and considering the ten-dimensional version of the solution. Since the circle is internal, the reduced type IIA string-frame metric takes the form of a stack of D2-branes

$$
\mathrm{d} s_{\mathrm{st}}^{2}=h^{-\frac{1}{2}} \mathrm{~d} x_{1,2}^{2}+h^{\frac{1}{2}}\left(\mathrm{~d} r^{2}+e^{2 f} \mathrm{~d} \Omega_{4}^{2}+e^{2 g}\left[\left(E^{1}\right)^{2}+\left(E^{2}\right)^{2}\right]\right),
$$

together with a slightly modified dilaton $e^{\Phi}=h^{\frac{1}{4}} e^{\Lambda}$. The vielbeins $E^{1}$ and $E^{2}$ describe a $\mathrm{S}^{2}$ fibration over $\mathrm{S}^{4}$, the latter with metric $\mathrm{d} \Omega_{4}^{2}$ (see appendix A.1 for the details). For fixed values of $e^{f}$ and $e^{g}$, the internal metric is that of $\mathbb{C P}^{3} \simeq \operatorname{Sp}(2) / \mathrm{U}(2)$, which is squashed with respect to the Fubini-Study metric whenever $e^{f} \neq e^{g}$. In the complete solution, this squashing changes with the radial direction and thus along the RG flow. The four-sphere coincides with the four-cycle that does not contract in the IR of the M-theory realisation. However, in ten dimensions the presence of the mass gap is obscured by the fact that the warp factor $h$ has an IR singularity.

The exact form of the metric functions can be found in [10] and will not be needed here. It can be seen that the UV of the entire family of geometries is that of $N$ coincident D2-branes in the decoupling limit

$$
e^{2 f}=2 e^{2 g} \sim r^{2}, \quad \quad e^{\Phi} \sim h^{\frac{1}{4}}, \quad h \sim N r^{-5} .
$$

The asymptotic value of the squashing, $e^{2 f-2 g}=2$, is fixed for all the solutions and corresponds to the nearly Kähler point of the $\mathbb{C P}^{3}$. The gauge theory dual for such metric was proposed in [17] and consists of a two-sites Yang-Mills quiver with $\mathrm{U}(N) \times \mathrm{U}(N)$ gauge group and bifundamental matter, similar to the Klebanov-Witten quiver in four dimensions. Additionally, since the circle on which we reduce from eleven dimensions is nontrivially fibered, we get a non-vanishing internal two-form, which generates Chern-Simons interactions in the gauge theory dual. Finally, there are also internal three- and four-form fluxes signalling the presence of fractional D2-branes. We expect these to correspond to a shift $M$ in the rank of one of the gauge groups. Thus, the conjecture is that these solutions are dual to RG flows in a

$$
\mathrm{U}(N)_{k} \times \mathrm{U}(N+M)_{-k}
$$

quiver gauge theory with CS interactions at level $k$ and preserving $\mathcal{N}=1$ supersymmetry. Given that the ten-dimensional metric is IR singular, in this setup the correct label for the different solutions is not the position of the end-of-space (equivalently the radius of the four-sphere at that point) but the asymptotic (UV) value of the NS two-form through a two-cycle inside $\mathbb{C P}^{3}$, which is in one-to-one correspondence with the former [10]. This parameter, that we call $b_{0}$, has the advantage of having a direct interpretation in the gauge theory dual as controlling the asymptotic difference between the microscopic Yang-Mills couplings of each of the two factors in the gauge group [18]

$$
b_{0} \sim \frac{1}{g_{1}^{2}}-\frac{1}{g_{2}^{2}} .
$$




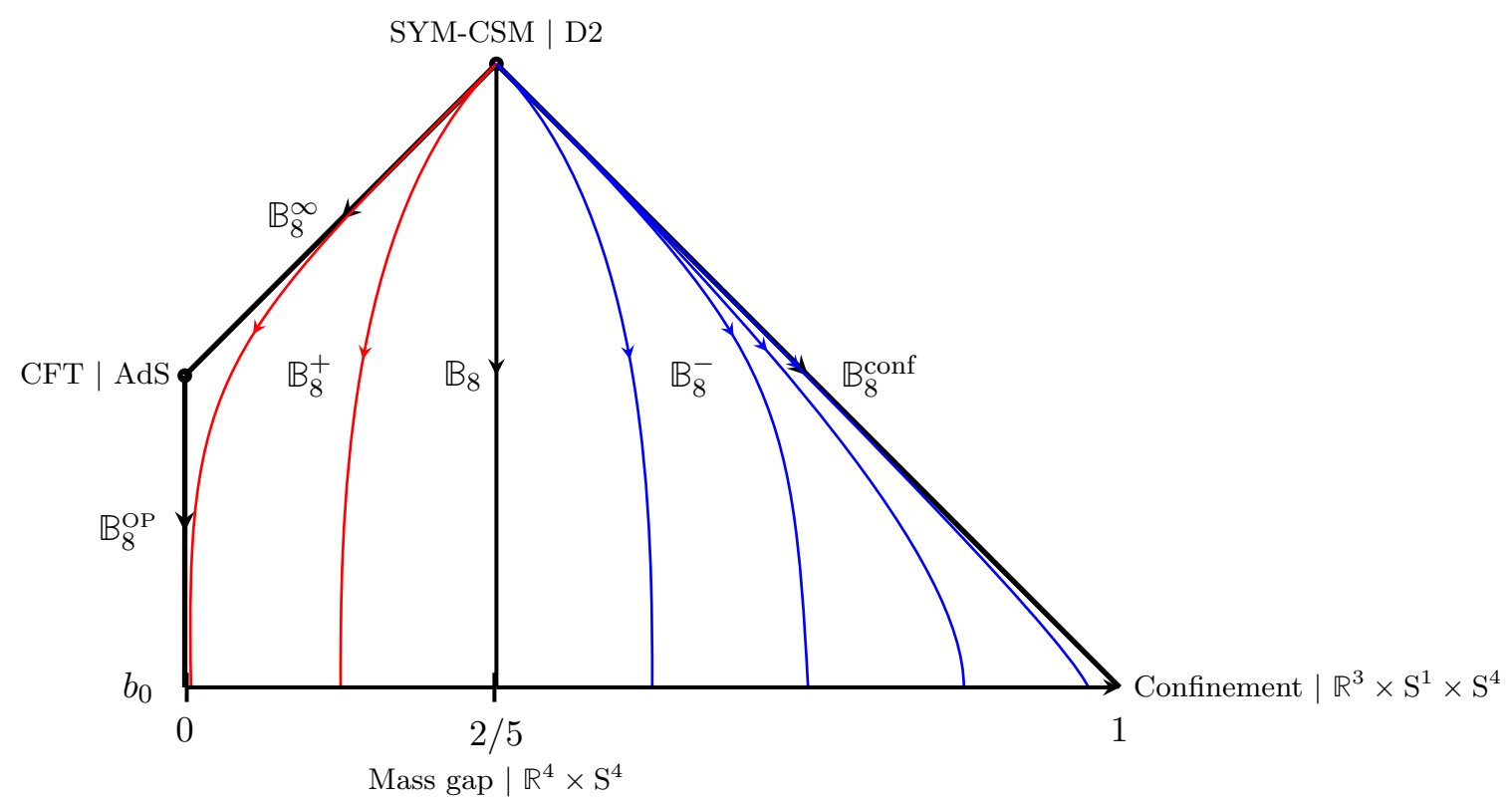

Figure 1. Pictorial representation of the different ground state solutions (see [10] for further details).

In our conventions $b_{0} \in[0,1]$. The two endpoints of the interval are special in that they lead to IR physics qualitatively different from the rest of the family. On the one hand, the solution with $b_{0}=1$, constructed originally in [19], is not only gapped but confining. This was attributed to the vanishing of the CS level in [10], in agreement with the arguments in [20]. We denote this solution $\mathbb{B}_{8}^{\text {conf }}$. On the other hand, when the difference between the couplings vanishes, $b_{0}=0$, the mass gap is lost and the theory flows to an IR fixed point described by the Ooguri-Park CFT [21]. This flow is denoted $\mathbb{B}_{8}^{\infty}$. Notice that for arbitrarily-small but non-vanishing values of $b_{0}$ the RG flow will pass arbitrarily close to the fixed point before reaching the gapped phase, giving rise to quasi-conformal dynamics in a certain range of energies. The setup is summarised in figure 1 , where we depict the different transverse geometries as a function of $b_{0}$, together with their IR limit and the associated gauge theory dual interpretation. A more detailed explanation can be found in [10].

This family of solutions can be straightforwardly heated up by going to Euclidean space and declaring the time direction to be compact. As usual, the period $\beta$ of the Euclidean time is related to the temperature $T$ in the dual gauge theory as

$$
\beta=\frac{1}{T} .
$$

The thermodynamic properties are very simple. The ground states are supersymmetric, so in the appropriate renormalisation scheme (see appendix A.2) the free energy $F$, computed as the bulk on-shell action, vanishes. Since formally these finite-temperature solutions coincide with the supersymmetric ones (they only differ globally in the time direction), their free energy will also vanish in the same scheme. This happens independently of the temperature, and therefore the entropy $S$ is also zero. 
These thermal states are continuously connected to the ground state, so we expect them to correspond to a phase still exhibiting a mass gap. On the other hand, we know that replacing the regular IR with a horizon also introduces temperature into the system while removing the gap. These solutions, describing black branes, can have the same UV asymptotics and therefore correspond to other finite-temperature states of the same gauge theories. In the following we construct the black brane solutions and show the existence of several phase transitions.

\section{High-temperature phases}

In this section we construct high-temperature phases of the gauge theories we have discussed. On the gravity side of the duality this corresponds to the replacement of the regular IR by a horizon, giving rise to a black brane. These black branes need to satisfy, at leading order, identical D2-brane UV boundary conditions as the zero-temperature solutions in order to correspond to states in the same gauge theory duals. Therefore we will pick the following ansatz for the type-IIA string frame metric and dilaton

$$
\begin{aligned}
\mathrm{d} s_{\mathrm{st}}^{2} & =h^{-\frac{1}{2}}\left(-\mathrm{b} \mathrm{d} t^{2}+\mathrm{d} x_{1}^{2}+\mathrm{d} x_{2}^{2}\right)+h^{\frac{1}{2}}\left(\frac{\mathrm{d} r^{2}}{\mathrm{~b}}+e^{2 f} \mathrm{~d} \Omega_{4}^{2}+e^{2 g}\left[\left(E^{1}\right)^{2}+\left(E^{2}\right)^{2}\right]\right), \\
e^{\Phi} & =h^{\frac{1}{4}} e^{\Lambda},
\end{aligned}
$$

which differs from that in eq. (2.1) only in the presence of a blackening factor $b$. This function must have a simple zero at the position of the horizon for the solution to describe a black brane.

The ansatz for the different fluxes takes the exact same form as for the supersymmetric solutions, as given in eq. (A.12). They are parametrised by three functions of the radial coordinate, $b_{J}, b_{X}$ and $a_{J}$, together with three constants $Q_{c}, q_{c}$ and $Q_{k}$. These constants are Page charges for D2, D4 and D6-branes and are thus quantised. Moreover, they are related to the gauge theory parameters appearing in eq. (2.3) as [10]

$$
Q_{c}=3 \pi^{2} \ell_{s}^{5} g_{s} N, \quad q_{c}=\frac{3 \pi \ell_{s}^{3} g_{s}}{4} \bar{M}, \quad Q_{k}=\frac{\ell_{s} g_{s}}{2} k,
$$

where we have defined the combination

$$
\bar{M}=\left(M-\frac{k}{2}\right) .
$$

In our conventions we must take $Q_{k}<0$.

These equations make explicit the length dimensions of the different charges. We can thus use them to shift and rescale the various functions of the ansatz as

$$
\begin{array}{rlrl}
e^{f} & =\left|Q_{k}\right| e^{\mathcal{F}}, & e^{g}=\left|Q_{k}\right| e^{\mathcal{G}}, & h=\frac{4 q_{c}^{2}+3\left|Q_{k}\right| Q_{c}}{\left|Q_{k}\right|} \mathbf{h}, \\
a_{J}=-\frac{q_{c}}{6}-\sqrt{4 q_{c}^{2}+3\left|Q_{k}\right| Q_{c}} \mathcal{A}_{J}, & b_{X}=\frac{2 q_{c}}{3\left|Q_{k}\right|}+\frac{\sqrt{4 q_{c}^{2}+3\left|Q_{k}\right| Q_{c}}}{3\left|Q_{k}\right|} \mathcal{B}_{X}, \\
b_{J}=-\frac{2 q_{c}}{3\left|Q_{k}\right|}-\frac{\sqrt{4 q_{c}^{2}+3\left|Q_{k}\right| Q_{c}}}{3\left|Q_{k}\right|} \mathcal{B}_{J}, &
\end{array}
$$


so that the redefined functions are dimensionless. Furthermore, working with the dimensionless radial coordinate

$$
u=\frac{\left|Q_{k}\right|}{r}
$$

all the charges drop from the equations. This means that, up to simple rescalings, the only parameter distinguishing one theory from another is $b_{0}$. In particular, the mass gap at zero temperature (in units of the 't Hooft couplings) is fixed by this parameter. The thermal phase transitions that we will exhibit will take place at specific values of the ratio $T / M_{\text {gap }}$ that are determined by $b_{0}$.

\subsection{Boundary conditions}

In the radial coordinate defined in eq. (3.5) the UV is located at $u=0$. The leading behaviour of the functions must coincide with that of the ground state. It is then possible to solve the equations order by order in the radial coordinate around this point, obtaining an expansion of the form

$$
\begin{array}{rlrl}
e^{\mathcal{F}} & =\frac{1}{u \sqrt{2}}\left[1+f_{1} u+\cdots+f_{4} u^{4}+f_{5} u^{5}+\cdots+f_{10} u^{10}+\mathcal{O}\left(u^{11}\right)\right], \\
e^{\mathcal{G}} & =\frac{1}{2 u}[1+\mathcal{O}(u)], & \mathbf{h} & =\frac{16}{15}\left(1-b_{0}^{2}\right) u^{5}[1+\mathcal{O}(u)], \\
\mathbf{b} & =1+\mathrm{b}_{5} u^{5}+\mathcal{O}\left(u^{6}\right), & e^{\Lambda} & =1+\mathcal{O}(u), \\
\mathcal{B}_{J} & =b_{0}+\cdots+b_{4} u^{4}+\cdots+b_{6} u^{6}+\cdots+b_{9} u^{9}+\mathcal{O}\left(u^{10}\right), \\
\mathcal{B}_{X} & =b_{0}+\mathcal{O}(u), & \mathcal{A}_{J} & =\frac{b_{0}}{6}+\mathcal{O}(u),
\end{array}
$$

where we have made explicit the parameters that remain undetermined by the equations of motion. The first coefficients not explicitly shown in this expansion, written in terms of the ones that are shown, can be found in appendix B. As we already argued, $b_{0}$ selects a particular member of the family of gauge theories. The radial gauge chosen in eq. (3.1), $g_{t t} g_{r r}=-1$, still leaves a residual freedom to shift the radial coordinate, $r \rightarrow r+$ constant. This allows us to fix the value of $f_{1}$ without loss of generality. We choose $f_{1}=-1$ in order to facilitate the comparison with the supersymmetric ground state. We are thus left with seven subleading, undetermined parameters in the UV. They correspond to normalisable modes and are hence related to the temperature (see eq. (3.9) below) and to vacuum expectation values for different operators in the dual gauge theory, including the energymomentum tensor. These parameters are fixed dynamically in the complete solution once we impose the presence of a regular horizon.

The existence of a horizon is encoded in a simple zero of the blackening factor $b$. At the horizon we demand regularity for the rest of the functions, so that they reach a finite value. We denote the position of the horizon as $u=u_{h}$ in the dimensionless radial coordinate introduced in eq. (3.5). In this way the functions enjoy an expansion of the form

$$
\begin{array}{rlrlrl}
e^{\mathcal{F}} & =f_{h}+\mathcal{O}\left(u-u_{h}\right), & e^{\mathcal{G}} & =g_{h}+\mathcal{O}\left(u-u_{h}\right), & e^{\Lambda} & =\lambda_{h}+\mathcal{O}\left(u-u_{h}\right), \\
\mathbf{h} & =h_{h}+\mathcal{O}\left(u-u_{h}\right), & \mathcal{B}_{J} & =\xi_{h}+\mathcal{O}\left(u-u_{h}\right), & \mathcal{B}_{X}=\chi_{h}+\mathcal{O}\left(u-u_{h}\right), \\
\mathcal{A}_{J} & =\alpha_{h}+\mathcal{O}\left(u-u_{h}\right), & \mathbf{b} & =\mathrm{b}_{h}\left(u-u_{h}\right)+\mathcal{O}\left(u-u_{h}\right)^{2} . & &
\end{array}
$$


All the subleading coefficients are determined in terms of these eight leading-order ones (see appendix $\mathrm{C}$ for the first coefficients). The position of the horizon $u_{h}$ controls the temperature of the black brane, so the phase diagram can be explored by changing its value.

\subsection{Numerical integration}

The perturbative analysis of the equations of motion with the desired boundary conditions in the UV and IR leaves us with the following undetermined parameters

$$
\begin{aligned}
\text { in the UV: } & f_{4}, f_{5}, f_{10}, b_{4}, b_{6}, b_{9}, \mathrm{~b}_{5} \\
\text { at the horizon: } & g_{h}, f_{h}, \lambda_{h}, h_{h}, \mathrm{~b}_{h}, \alpha_{h}, \xi_{h}, \chi_{h} .
\end{aligned}
$$

For each value of $b_{0}$ and $u_{h}$ we must find these fifteen parameters dynamically in the complete solution. Since we are solving eight second-order equations subject to one firstorder (Hamiltonian) constraint, the problem is well posed. It should be mentioned that, as we note in eq. (A.29), there is a conserved quantity along the radial coordinate. When substituting the expansions, this conserved quantity relates one of the UV parameters with horizon data as

$$
\mathrm{b}_{5}=\frac{16}{5} \frac{\mathrm{b}_{h} f_{h}^{4} g_{h}^{2}}{\lambda_{h}^{2}} u_{h}^{2}
$$

This equation can be used as a check for the numerical solutions. Alternatively, it can be imposed ab initio, reducing the number of parameters to be found numerically to fourteen.

The solutions were constructed using a shooting method. The boundary conditions, eqs. (3.6) and (3.7), were imposed close to the UV and the horizon, respectively, with an initial guess for the unfixed parameters, giving us a seed solution. The full equations were then integrated numerically both from the UV and from IR up to a designated intermediate matching point. For arbitrary values of the parameters the functions would be discontinuous at that point. Starting from this seed, we then used the Newton-Raphson algorithm to find the values of the parameters such that the functions and their derivatives are continuous at the matching point with the desired precision.

The main challenge when solving a system of equations via the shooting method is to find a good initial seed. Ultimately, we are using a Newton-Raphson algorithm on a fourteen-dimensional parameter space (after making use of eq. (3.9)), so we need to start the search close enough to the correct values of the unknown constants for it to converge. A good seed for a solution can be provided by another solution close enough in parameter space since, by continuity, the values of the unknown constants will be similar. In other words, once we have a black brane with given control parameters $b_{0}$ and $u_{h}$, we can use the values of the rest of the parameters as the initial guess for another solution with slightly corrected control parameters. The problem thus reduces to finding the first black brane.

Fortunately the system admits one analytic solution describing a black brane. As we already mentioned, the RG flow corresponding to $b_{0}=0$, denoted by $\mathbb{B}_{8}^{\infty}$ at the left of figure 1, ends at an IR fixed point. This is located at $r=-\pi Q_{k}$ (equivalently, $u=\pi^{-1}$ ) 
so that near this point the functions in the metric read

$$
\begin{aligned}
e^{2 f} & =\frac{9}{5}\left(r+\pi Q_{k}\right)^{2}, & e^{2 g} & =\frac{9}{25}\left(r+\pi Q_{k}\right)^{2}, \\
e^{\Lambda} & =\frac{3}{5\left|Q_{k}\right|}\left(r+\pi Q_{k}\right), & h & =\frac{125 Q_{k}^{4}}{2187\left(r+\pi Q_{k}\right)^{4}},
\end{aligned}
$$

giving rise to an $\mathrm{AdS}_{4}$ geometry, as expected. It is then straightforward to include a horizon by considering the blackening factor

$$
\mathrm{b}(r)=1-\frac{\left(r_{h}+\pi Q_{k}\right)^{3}}{\left(r+\pi Q_{k}\right)^{3}},
$$

which describes an AdS-Schwarzschild black brane. This is an exact solution to the equations of motion when $b_{0}=0$.

Based on this observation, the strategy to find the first black brane with the desired asymptotics is the following. We put a horizon in the region where the geometry is close to AdS-Schwarzschild and we choose as horizon data the values of the functions for $\mathbb{B}_{8}^{\infty}$ at that point. Since this should correspond to a solution at low temperature, the UV parameters are then taken as the ones for the metric without a horizon. Also, we use eq. (3.11) as the seed for the blackening factor at both the UV and at the horizon. As a final simplification, we note that for $b_{0}=0$ the dimensionless functions $\mathcal{B}_{J}, \mathcal{B}_{X}$ and $\mathcal{A}_{J}$ from eq. (3.4) vanish exactly and, consequently, there are three equations which are trivially satisfied and six fewer parameters to be found. In this way we were able to find a black brane solution in the $\mathbb{B}_{8}^{\infty} \mathrm{RG}$ flow, with the horizon located at $u_{h} \approx \pi^{-1}-4 \times 10^{-4}$.

Once we have the first black brane we slightly decrease $u_{h}$ and use as seed the values of the parameters we have just obtained, finding in this way another solution. Thus, we can iteratively find hotter black branes by interpolating the values of the parameters of the previous solutions to obtain a good seed for the next one. The resulting family of black branes has the expected properties. At low temperatures the dependence of the free energy on the temperature is dictated by conformal invariance,

$$
F \sim \frac{1}{|k|}\left(\frac{\bar{M}^{2}}{2}+N|k|\right)^{3 / 2} T^{3} .
$$

In the opposite, UV-limit, for temperatures above

$$
\lambda \frac{k^{2}}{N}\left(\frac{\bar{M}^{2}}{2}+N|k|\right)^{-1 / 2}
$$

with $\lambda=g_{s} \ell_{s}^{-1} N$ denoting 't Hooft's coupling, we recover the D2-brane solution and the free energy behaves as in a three-dimensional Yang-Mills theory at strong coupling:

$$
F \sim \lambda^{-1 / 3}\left(\frac{N}{|k|^{5}}\right)^{1 / 3}\left(\frac{\bar{M}^{2}}{2}+N|k|\right)^{5 / 3} T^{10 / 3}
$$


This UV behaviour will be shared by the entire family of solutions. Note that in the $N \rightarrow \infty$ limit the free energy scales with the number of colours as $N^{2}$, as expected on a deconfined phase.

Taking into account that all the flows share the same UV asymptotics except for the value of $b_{0}$, we can use the solutions we just constructed as a seed to find black branes with non-vanishing $b_{0} \gtrsim 0$. The seed will be better the higher the temperature is, since the flows differ mostly in the IR. Now $\mathcal{B}_{J}, \mathcal{B}_{X}$ and $\mathcal{A}_{J}$ are also non-vanishing, hence we have to solve the full system of equations and shoot for the complete set of parameters. In this way we obtained the first high-temperature black brane for a small non-vanishing value of $b_{0}$, whose ground state is gapped. Again, using this first solution as a seed it is possible to gradually increase the value of $u_{h}$ and find colder black branes. The process ends when some of the functions, namely $e^{\mathcal{F}}, e^{\mathcal{G}}$ and $e^{\Lambda}$, vanish at the horizon within numerical precision. We will refer to the value of $u_{h}$ where this happens as $u_{N}$, which is a meaningful quantity because we have fixed the radial gauge completely. In the case of the zero-temperature, regular solutions the vanishing of these metric functions is precisely what produces the end-of-space and the mass gap. Below we will investigate whether this happens in the current context in a smooth or in a singular manner.

Once we have the entire family of black branes for this particular $b_{0}$, we slightly increase its value. The hottest black branes would again be similar to the ones we had constructed, that may then be used as a seed. With this strategy it is possible to explore the entire space of parameters in the $\left(b_{0}, u_{h}\right)$-plane. Plots for some of the solutions can be found in appendix D.

\subsection{Zero-entropy limit}

Before moving into the study of the phase diagram, let us discuss in more detail the solutions that we obtain in the limit in which we remove the horizon for the black brane solutions with $0<b_{0}<1$. This is achieved when the values of the functions $e^{\mathcal{F}}, e^{\mathcal{G}}$ and $e^{\Lambda}$ become zero at $u_{h}=u_{N}$, since the vanishing of these functions implies that the area of the black branes, and therefore their entropy, goes to zero. As we will see, in this limit the temperature is still non-zero.

As we have defined it, $u_{N}$ is the maximum value fo $u_{h}$ for a given $b_{0}$. The first observation is that this does not coincide with $u_{s}\left(b_{0}\right)$, defined as the position of the end-ofspace for the supersymmetric, regular, zero-temperature solution with the same $b_{0}$. Note that it is meaningful to compare these two coordinate values because we have fixed the radial gauge completely. The discrepancy can be seen for the particular example of $b_{0}=2 / 5$ in figure 2, where we show the value of $e^{\mathcal{F}}, e^{\mathcal{G}}$ and $e^{\Lambda}$ at the horizon as a function of the position of the horizon normalized to $u_{s}$. All three values vanish at the same point $u_{N}<u_{s}$. The same phenomenon can be seen for different values of $b_{0}$ in appendix D.

As a consequence, when we take the area of the horizon to zero, which we refer to as the "zero-entropy limit," we do not recover the ground state solutions discussed in section 2. This is in sharp contrast with well known examples such as AdS-Schwarzschild or black Dp-branes, where the usual AdS and Dp-brane metrics are recovered as one removes the 


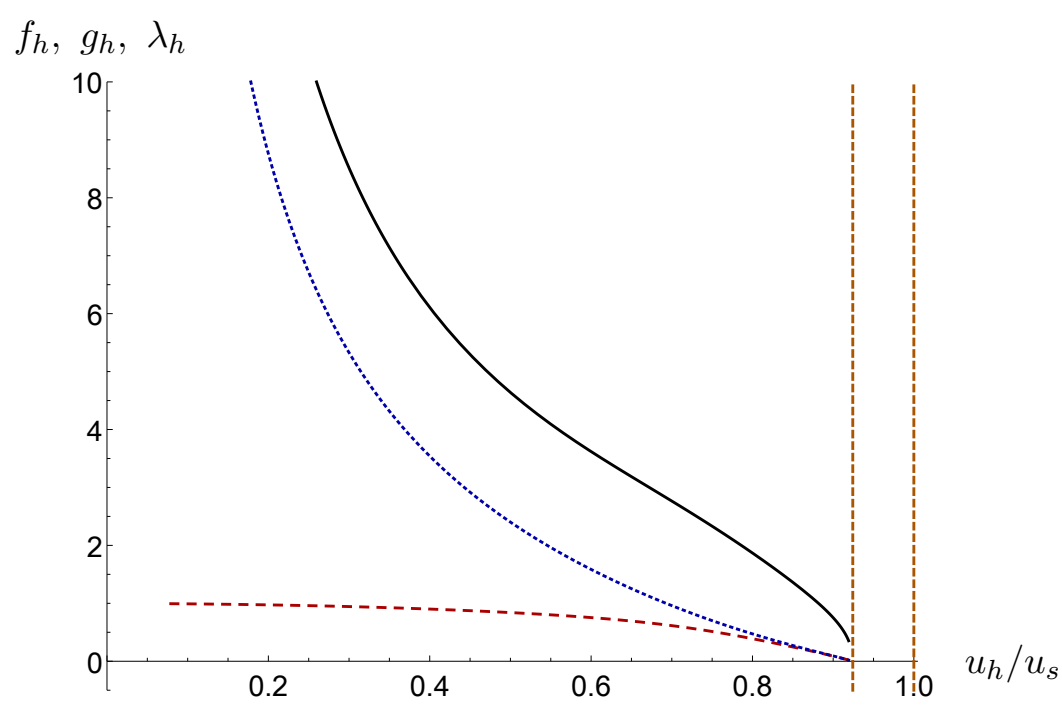

Figure 2. Horizon values of $e^{\mathcal{F}}, e^{\mathcal{G}}$ and $e^{\Lambda}$ in solid black, dotted blue and dashed red, respectively, as a function of the position of the horizon normalized to $u_{s}$, which is the where the supersymmetric regular ground-state solution ends. The dashed orange vertical line to the left corresponds to $u_{N}$, while the one to the right represents $u_{s}$. Between those two lines we have not found any black brane solutions. In this plot, we have fixed $b_{0}=2 / 5$.

horizon. It is therefore interesting to understand the nature of the solution obtained in this regime.

One indication comes from the fact that the eleven-dimensional curvature at the horizon diverges as we remove it. This suggests that a naked singularity is uncovered in this limit. This is illustrated in figure 3, where we show the Ricci scalar in terms of the horizon value of $e^{\Lambda}, \lambda_{h}$, in order to obtain a manifestly gauge-invariant plot. It can be seen that the scalar curvature at the horizon diverges as $R \sim \lambda_{h}^{-2 / 3}$ in the limit $\lambda_{h} \rightarrow 0$, that is, as $u_{h} \rightarrow u_{N}$. This behaviour is of course inherited from that of the metric functions. Indeed, it is possible to infer their dependence on $\lambda_{h}$ near zero from the numerics. For the internal components we obtain

$$
e^{\mathcal{F}} \sim \lambda_{h}^{1 / 2}, \quad e^{\mathcal{G}} \sim \lambda_{h}
$$

It turns out that this is precisely their behaviour in the IR of the $\mathbb{B}_{8}$ family, which is generically $\mathbb{R}^{4} \times \mathrm{S}^{4}$ (see figure 1 ). The transverse geometries in eleven dimensions ${ }^{1}$ obtained by removing the horizon are thus perfectly regular, capping off smoothly at a certain $u_{N}$. Furthermore, the coincidence with a $\mathbb{B}_{8}$ metric goes beyond the IR and extends to the entire solution. In figure 4 (top) we compare the metric functions that describe the transverse metric for the solution with lowest entropy that we constructed for $b_{0}=2 / 5$ with those of the $\mathbb{B}_{8}$ metric that ends at the same value of $u_{N}$. They coincide within numerical precision except in a tiny IR region due to the small horizon that still exists in the thermal solution. We conclude that, as far as the eight-dimensional transverse geometry is concerned, the

\footnotetext{
${ }^{1}$ As given by eq. (A.15), with the metric functions found numerically.
} 


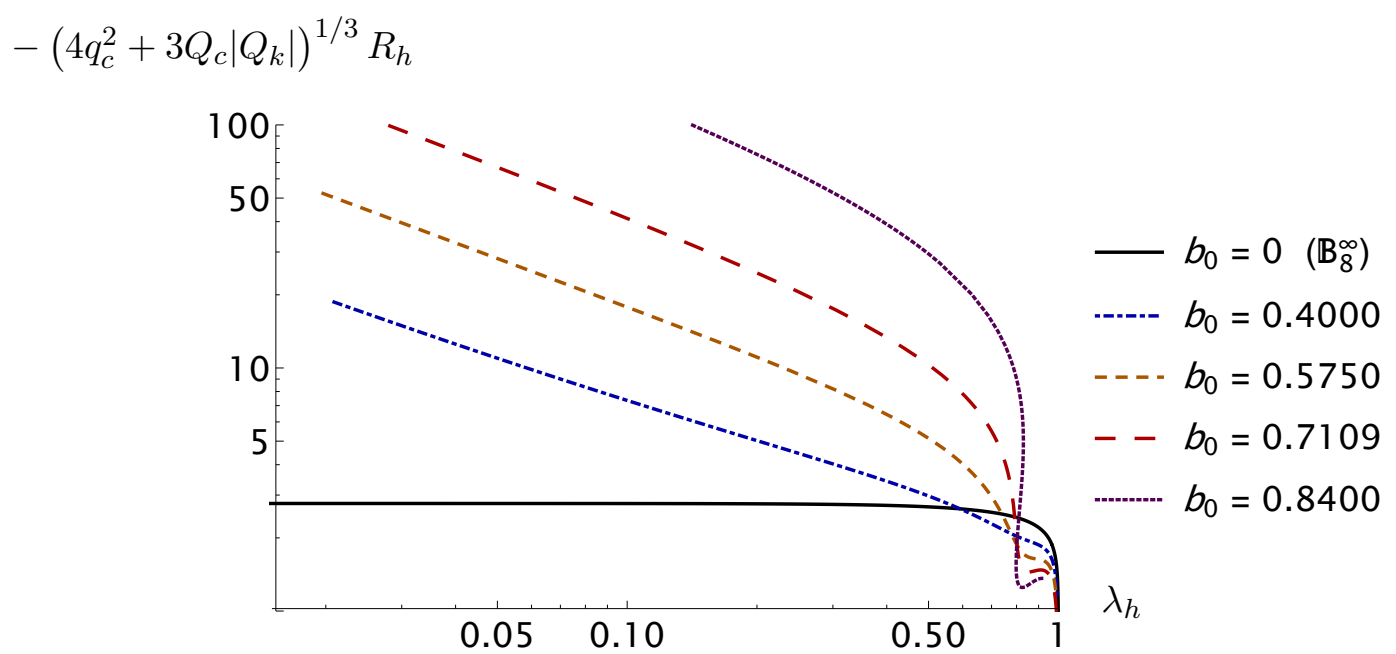

Figure 3. Eleven-dimensional Ricci scalar as a function of $e^{\Lambda}$, both evaluated at the horizon, for different black brane solutions. Notice that as $\lambda_{h}$ goes to zero (i.e. in the zero-entropy limit), the Ricci scalar diverges for a generic value of $b_{0}>0$, whereas for $\mathbb{B}_{8}^{\infty}$ it approaches the constant value corresponding to the IR AdS. The curvature invariants obtained by squaring the Ricci and Riemann tensors have an analogous behaviour.

zero-entropy limit $u_{h} \rightarrow u_{N}$ describes a regular $\mathbb{B}_{8}$ metric with the end-of-space at $u_{N}$ instead of the $u_{s}$ of the corresponding ground state.

The singularity in the full eleven-dimensional metric must then come from the warp factor. As we approach the zero-entropy limit it goes as

$$
\mathbf{h} \sim \lambda_{h}^{-2} .
$$

Again, this behaviour can be identified with that of a known solution. As explained in section 2, for the horizonless solutions to be regular it is necessary to include internal fluxes (describing fractional branes) with fine-tuned values. If these values are not the correct ones the warp factor is IR-singular, with a divergence given exactly by eq. (3.16). We thus conclude that the zero-entropy limit of our black-brane solutions is a supersymmetric solution with fluxes, but the limiting values of these fluxes are not the correct ones to render the solution regular in the IR. This can be seen in figure 4 , where we compare, for $b_{0}=2 / 5$, the lowest-entropy black brane solution that we constructed (i) with the supersymmetric solution to the BPS equations in [10] with the end-of-space at $u_{N}(2 / 5)$, and (ii) with the regular supersymmetric ground state. The first two backgrounds agree within numerical precision except in a tiny IR region due to the impossibility of reaching $u_{h} \rightarrow u_{N}$ numerically, while the regular one is clearly distinct.

In summary, in the limit in which we remove the horizon and the entropy vanishes we find a supersymmetric solution which is nevertheless singular, since the subleading coefficients that we recover are not the ones that ensure regularity of the ground state. The singularity is good in the sense of [28] because it can be hidden behind a horizon. It is interesting that this limit of zero entropy is reached at a finite temperature, as we will see in detail in the next section. 

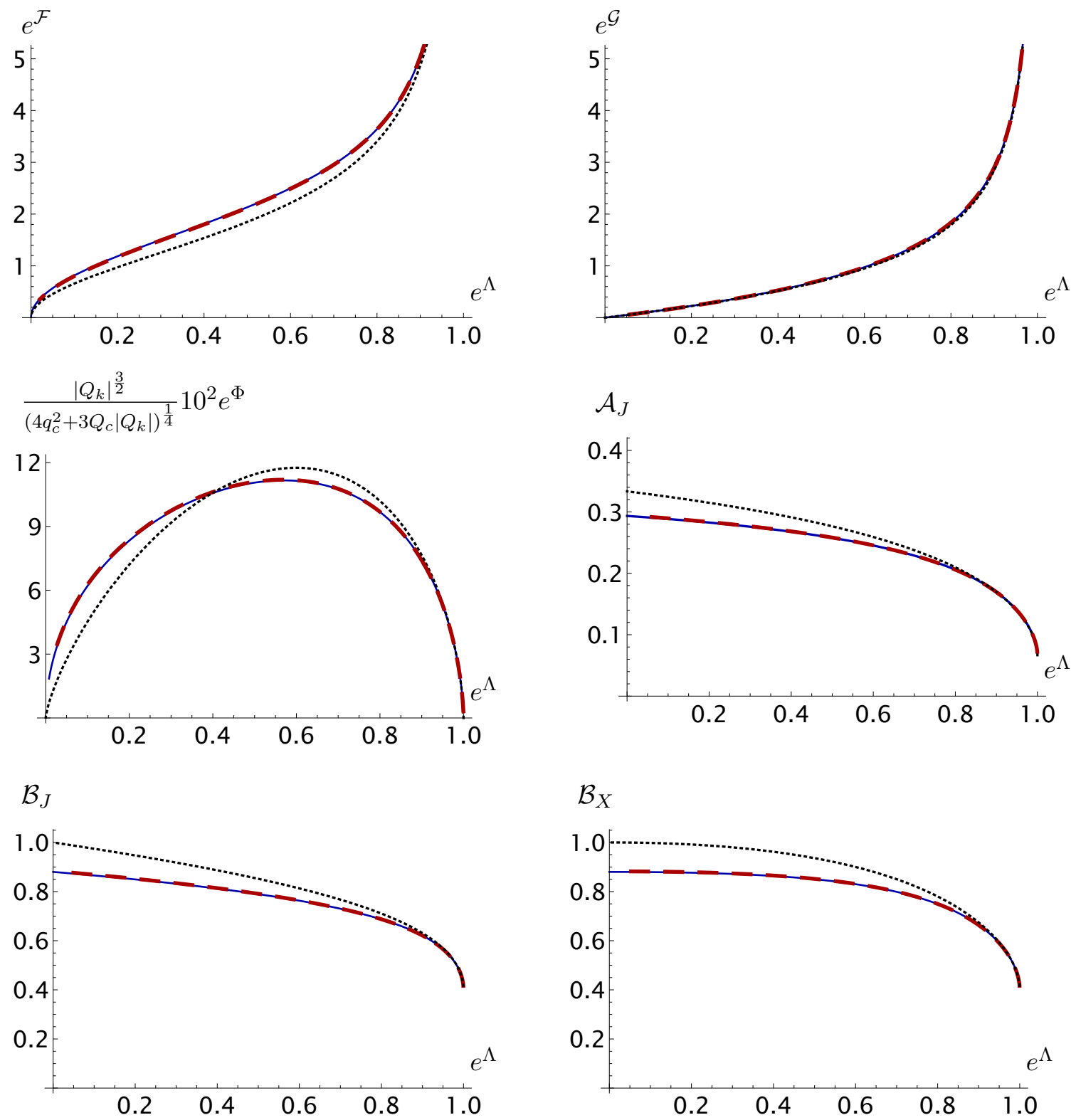

Figure 4. Comparison of three solutions with $b_{0}=2 / 5$. The red, dashed curves correspond to the lowest-entropy solution that we were able to construct. The solid, blue curves show the horizonless, supersymmetric singular solution. These two solutions only differ in a tiny IR region. The dotted, black curves correspond to the horizonless, regular solution. 


\section{Thermodynamics and the phase diagram}

In this section we will discuss the main thermodynamic properties of the black branes we have constructed and confirm the presence of phase transitions between themselves and between them and the low-temperature phases described in section 2. In this way we will construct the phase diagram in the $\left(b_{0}, T\right)$-plane for the entire family of theories.

\subsection{Thermodynamic quantities}

The renormalized four-dimensional bulk action $S_{\text {ren }}$ describing the system is obtained in section A.2. From this it is possible to extract the different thermodynamic quantities. The free energy density is given by

$$
F=-\frac{S_{\text {ren }}}{\beta V_{2}}
$$

with $V_{2}$ the (infinite) volume in the spatial directions and $\beta$ the period of the compact Euclidean time. This period, which is related to the temperature through eq. (2.5), is fixed in the black-brane geometries by requiring the absence of conical singularities. As usual the Bekenstein-Hawking entropy is given by the area of the horizon. Using the known UV and horizon expansions, eqs. (3.6) and (3.7), to evaluate these expressions, we obtain the following dimensionless values for the free energy, entropy and temperature

$$
\begin{aligned}
& \bar{F}=\frac{2 \kappa_{4}^{2}}{\left|Q_{k}\right|^{5}} F=-\frac{411}{2}-6 f_{4}-2 f_{5}+\frac{3}{2} \mathrm{~b}_{5}, \\
& \bar{S}=\frac{2 \kappa_{4}^{2}}{\left|Q_{k}\right|^{3}\left(4 q_{c}^{2}+3 Q_{c}\left|Q_{k}\right|\right)^{1 / 2}} S=\frac{64 \pi f_{h}^{4} g_{h}^{2} \sqrt{h_{h}}}{\lambda_{h}^{2}}, \\
& \bar{T}=\frac{\left(4 q_{c}^{2}+3 Q_{c}\left|Q_{k}\right|\right)^{1 / 2}}{\left|Q_{k}\right|^{2}} T=-\frac{1}{4 \pi} \frac{\mathrm{b}_{h} u_{h}^{2}}{\sqrt{h_{h}}},
\end{aligned}
$$

where $b_{5}$ is understood to be given by eq. (3.9) in terms of horizon data. Similarly, the internal energy density $E$ and pressure $P$, computed from the energy-momentum tensor in eq. (A.25), are given by

$$
\frac{2 \kappa_{4}^{2} P}{\left|Q_{k}\right|^{5}}=\frac{411}{2}+6 f_{4}+2 f_{5}-\frac{3}{2} \mathrm{~b}_{5}, \quad \bar{E}=\frac{2 \kappa_{4}^{2} E}{\left|Q_{k}\right|^{5}}=-\frac{411}{2}-6 f_{4}-2 f_{5}-\frac{7}{2} \mathrm{~b}_{5},
$$

which fulfil the expected thermodynamic relations $F=-P$ and $F=E-T S$. Finally, it must be verified that

$$
S=-\frac{\mathrm{d} F}{\mathrm{~d} T},
$$

which can be used as a check of the numerical results. Other quantities like the specific heat and the speed of sound can be straightforwardly computed from these thermodynamic potentials as:

$$
c_{v}=\frac{\mathrm{d} E}{\mathrm{~d} T}, \quad c_{s}^{2}=\frac{\mathrm{d} P}{\mathrm{~d} E}=\frac{S}{c_{v}}=\frac{S}{T} \frac{\mathrm{d} T}{\mathrm{~d} S} .
$$




\subsection{Phase diagram}

For each value of $b_{0}$, namely for each gauge theory in the family, we must determine the preferred solution at each temperature. The first type of solutions that compete are those of section 2, which are obtained from the horizonless, supersymmetric, regular solutions simply by compactifying the Euclidean time. The period of this thermal circle is arbitrary, so these geometries exist for any temperature and, in our renormalization scheme, they have vanishing free energy and entropy. The second type of solutions are the black branes found in section 3. Their free energy and entropy are given in terms of UV and horizon data by eq. (4.2) and have a non-trivial dependence on the temperature. If several solutions exist at a given temperature, the one with lower free energy will be thermodynamically preferred. Since the free energy of the first type of solutions vanishes, the black branes will dominate if their free energy is negative. At points where the free energy of black branes crosses zero there is a phase transition to the gapped state. The nature of the phase transition depends on the value of $b_{0}$. We identified two special values of $b_{0}$ at which the qualitative features change:

$$
b_{0}^{\text {critical }} \approx 0.6815, \quad b_{0}^{\text {triple }} \approx 0.6847 .
$$

Note that these are very close to one another. We will come back to this in section 5 . These two values give rise to the following three regions:

Case A: Phase transitions for $\boldsymbol{b}_{\mathbf{0}} \in\left(\boldsymbol{b}_{\mathbf{0}}^{\text {triple }}, \mathbf{1}\right]$. In this range the situation is similar to the typical Hawking-Page phase transition. The free energy crosses the horizontal axis at some critical temperature $T_{c}$. The preferred phase above $T_{c}$ is the black brane and below $T_{c}$ it is the horizonless, regular solution. Therefore at $T_{c}$ there is a phase transition between a gapped and an ungapped phase. At the critical temperature the entropy density jumps between some finite value and zero, signaling that the transition is first-order. In figure 5 we show the free energy and the entropy densities as a function of temperature for a representative case with $b_{0}=0.7902$.

Case B: Phase transitions for $b_{0} \in\left(b_{0}^{\text {critical }}, b_{0}^{\text {triple }}\right)$. In this small range the theories exhibit two phase transitions. This is illustrated in figure 6 , where we show the free energy and the entropy of the black branes for the theory with $b_{0}=0.6835$. Decreasing the temperature from asymptotically high values we first find a first-order phase transition between two black brane geometries at which the entropy density changes discontinuously. Therefore this transition takes place between two ungapped phases and is indicated in figure 6 by the dashed, red line. Decreasing the temperature further along the low-temperature black-brane branch we find that the entropy and the free energy densities vanish at a finite value of the temperature. In this zeroentropy limit we recover the horizonless, singular solution described in section 3.3. Strictly speaking, since this solution is singular, its temperature is undetermined. However, the limit along the regular branch of black brane solutions results in a fixed, non-zero value of the temperature, indicated by the black dot in figure 6 . At this temperature we expect a transition between the supersymmetric, horizonless, 

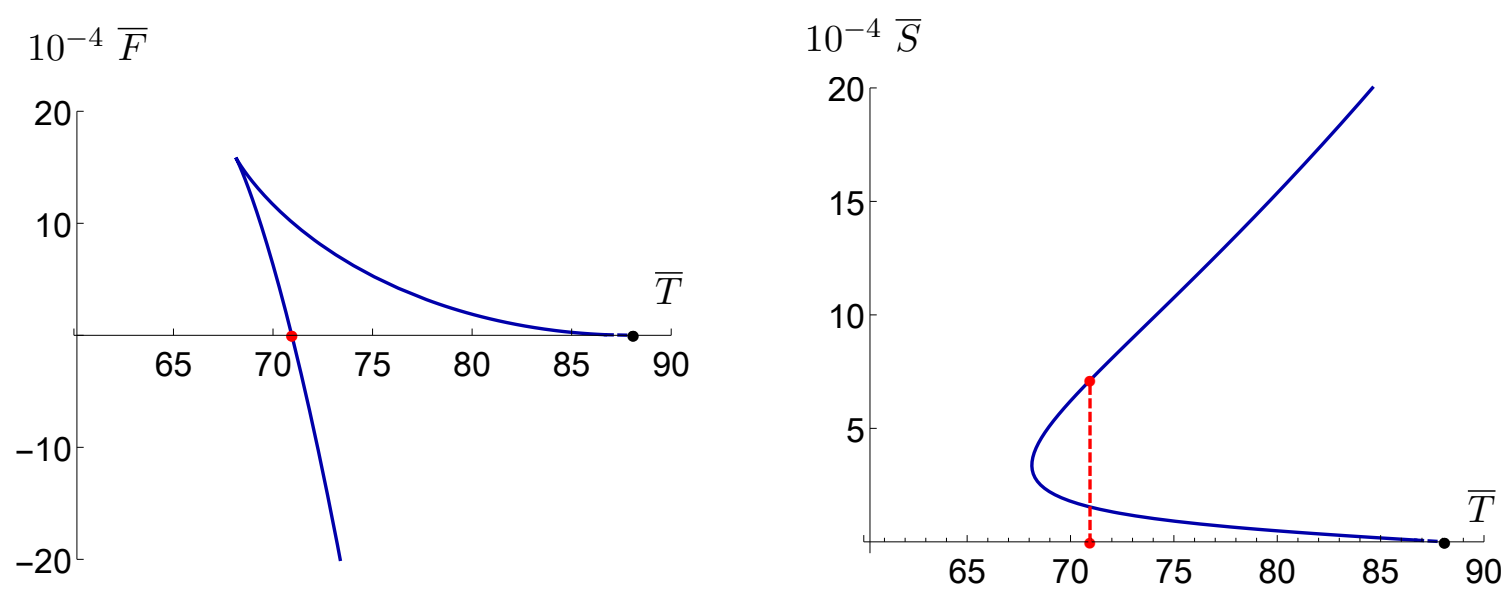

Figure 5. Free energy density (left) and entropy density (right) as a function of the temperature for black branes in the theory with $b_{0}=0.7902$ (Case A). When the free energy crosses the axis, there is a first-order phase transition at which the entropy changes discontinuously. The dashed, red line indicates the critical temperature. The region close to the horizontal axis of the blue curves, shown with dashes, is the result of an extrapolation to zero entropy.

singular solution and the supersymmetric, horizonless, regular solution with the same value of $b_{0}$. This transition is analogous to that in Case A except for the fact that the entropy density is continuous across the transition. Nevertheless, the transition is still first-order because the solution changes discontinuously, as illustrated in figure 4 . In the gauge theory this would be reflected in a discontinuity in observable quantities such as $n$-point functions. Note that, strictly speaking, the supergravity description breaks down sufficiently close to the transition since the curvature diverges at that point, as shown in figure 3.

Case C: Phase transitions for $\boldsymbol{b}_{\mathbf{0}} \in\left(\mathbf{0}, \boldsymbol{b}_{\mathbf{0}}^{\text {critical }}\right)$. In this range of values the phase transition between black branes disappears. The qualitative behaviour of the free energy and entropy densities is similar to that in figure 7 , where we show the results for $b_{0}=0.5750$. For the theories in this range there is a unique phase transition between the ungapped black brane phase and the regular gapped solution. Again, this happens at zero entropy but finite temperature, as in Case B.

The change from one behaviour to another happens smoothly as we vary $b_{0}$. Essentially, Case B is an intermediate scenario between Cases A and C. This can be seen by plotting the free energy and entropy densities for different values of the parameter in a small region that contains that of Case B entirely. The result is shown in figures 8 and 9 , respectively. The largest value of the parameter in the bottom panel of figure 8 is $b_{0} \approx 0.6861 \gtrsim b_{0}^{\text {triple }}$ and corresponds to the rightmost curve. As we decrease $b_{0}$, a small triangle, ending on the axis, starts to grow below the unstable branch. The lower side of this triangle is thermodynamically stable but not dominant. Decreasing further the parameter $b_{0}$, the lower side of the triangle ends up crossing the previous stable branch and becomes favoured. This explains the change from Case A to Case B. At this point the system goes from having 

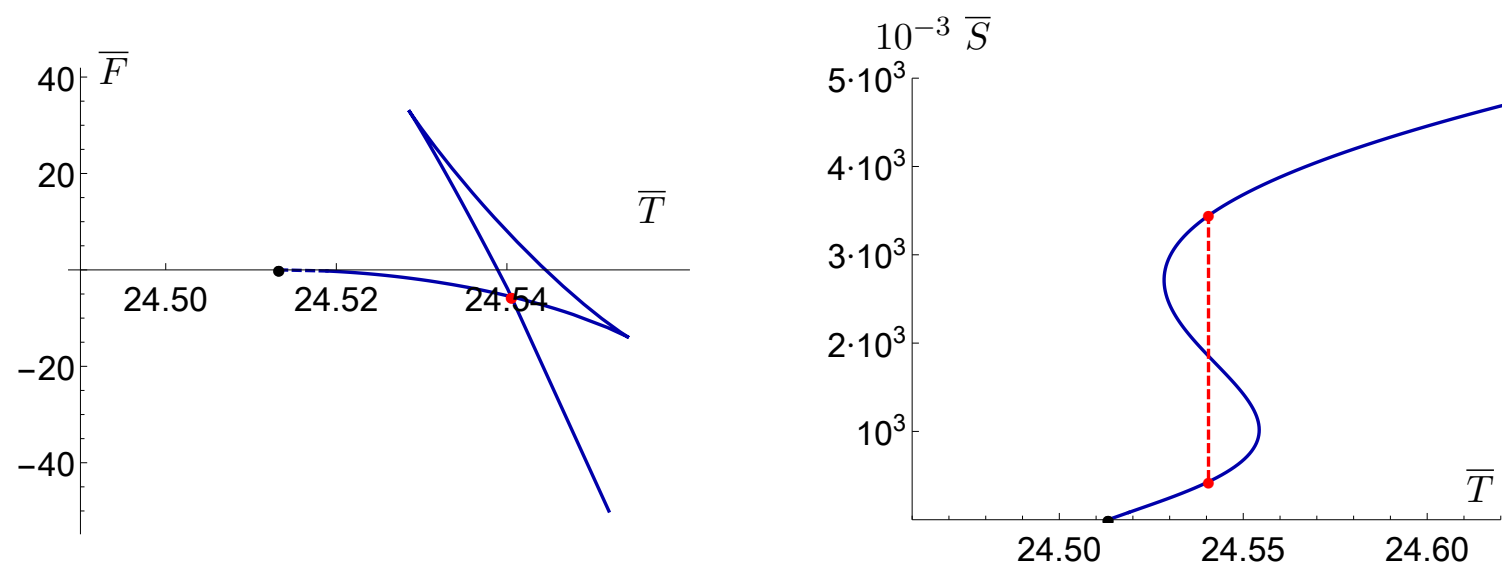

Figure 6. Free energy (left) and entropy (right) as a function of the temperature for black branes in the theory with $b_{0}=0.6835$ (Case B). As the temperature decreases, there is a first order phase transition between two branches of black branes. At the transition the entropy changes from some finite value to another finite value, represented by the dashed red line. Decreasing further the temperature, one would find another phase transition from the second black brane phase to the regular phase without a horizon. This happens at some finite value of the temperature and vanishing entropy. The region close to the horizontal axis of the blue curve, shown with dashes, is the result of an extrapolation to zero entropy.
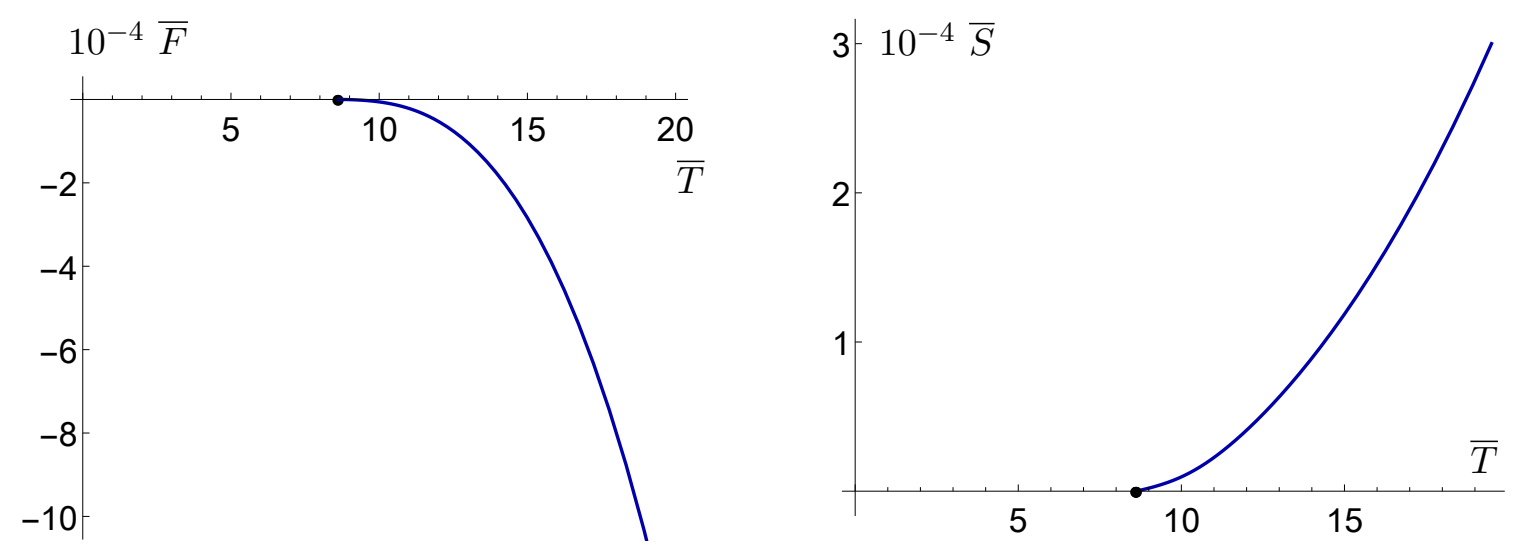

Figure 7. Free energy density (left) and entropy density (right) as a function of the temperature for black branes in the theory with $b_{0}=0.5750$ (Case C). In this example, there is a single phase transition that takes place at finite temperature and zero entropy. The region close to the horizontal axis of the blue curve, shown with dashes, is the result of an extrapolation to zero entropy.

one first-order transition to having two of them. Therefore this is a triple point at which three phases can coexist.

As the size of the second stable branch increases, the size of the triangle decreases. This explains the change from Case $\mathrm{B}$ to Case $\mathrm{C}$ : by reducing $b_{0}$ the triangle eventually disappears. This can be seen in the top panel of figure 8 , where the leftmost curve corresponds to $b_{0} \approx 0.6813 \lesssim b_{0}^{\text {critical }}$. The point where the triangle disappears is a critical point, namely the end of a line of first-order transitions. At this point the transition becomes second-order, as can be seen in the plot of the entropy density of figure 9. With all this 

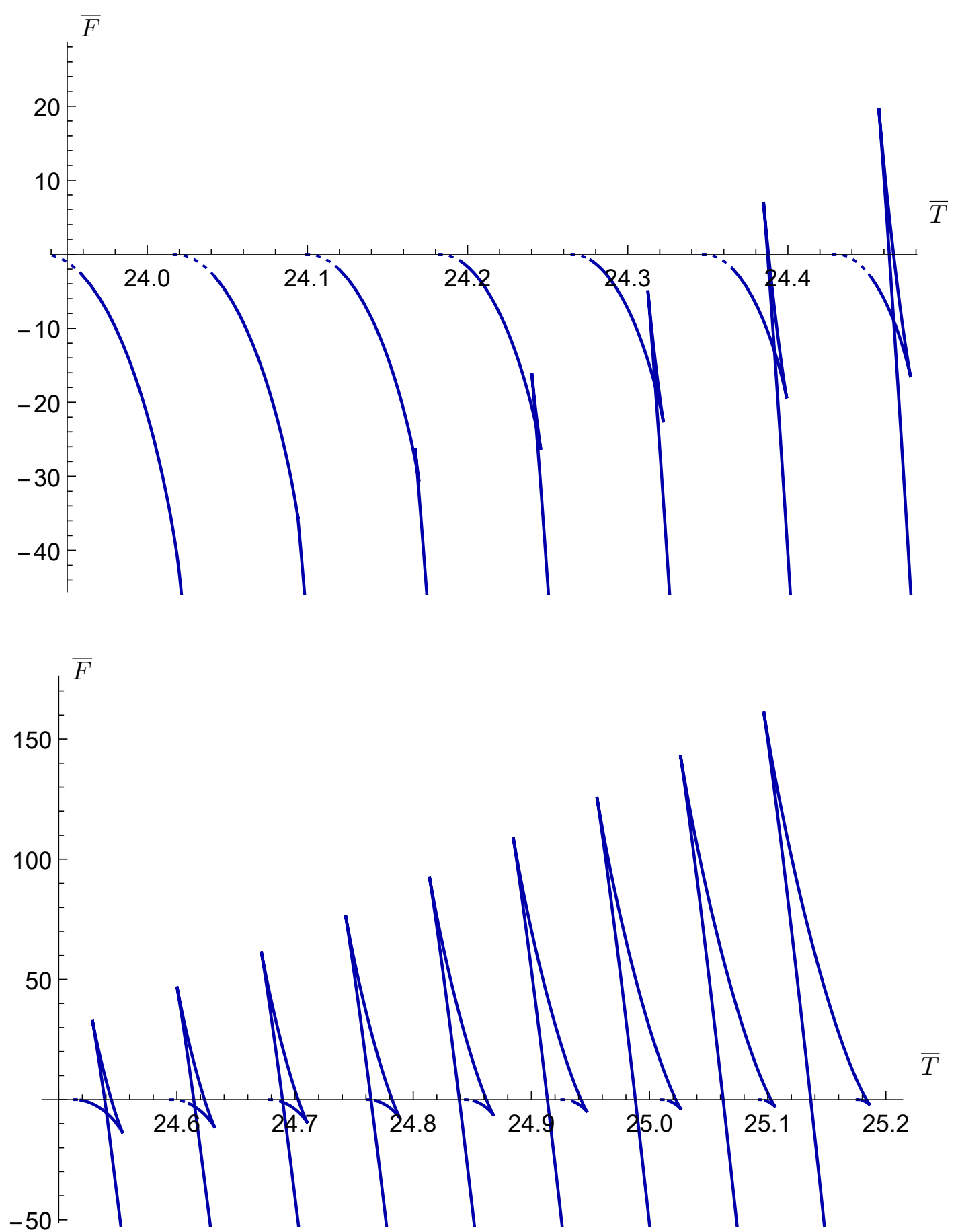

Figure 8. Free energy density for different theories with $b_{0}$ varying from $b_{0}=0.6813 \lesssim b_{0}^{\text {critical }}$ to $b_{0}=0.6861 \gtrsim b_{0}^{\text {triple }}$ from left to right and from top to bottom, with an approximate separation of $\Delta b_{0} \approx 0.0003$ between adjacent curves. A triangle develops as we decrease $b_{0}$ (bottom panel). If $b_{0}$ is decreased further then the triangle disappears (top panel). The region close to the horizontal axis of the curve, shown with dashes, is the result of an extrapolation to zero entropy. 


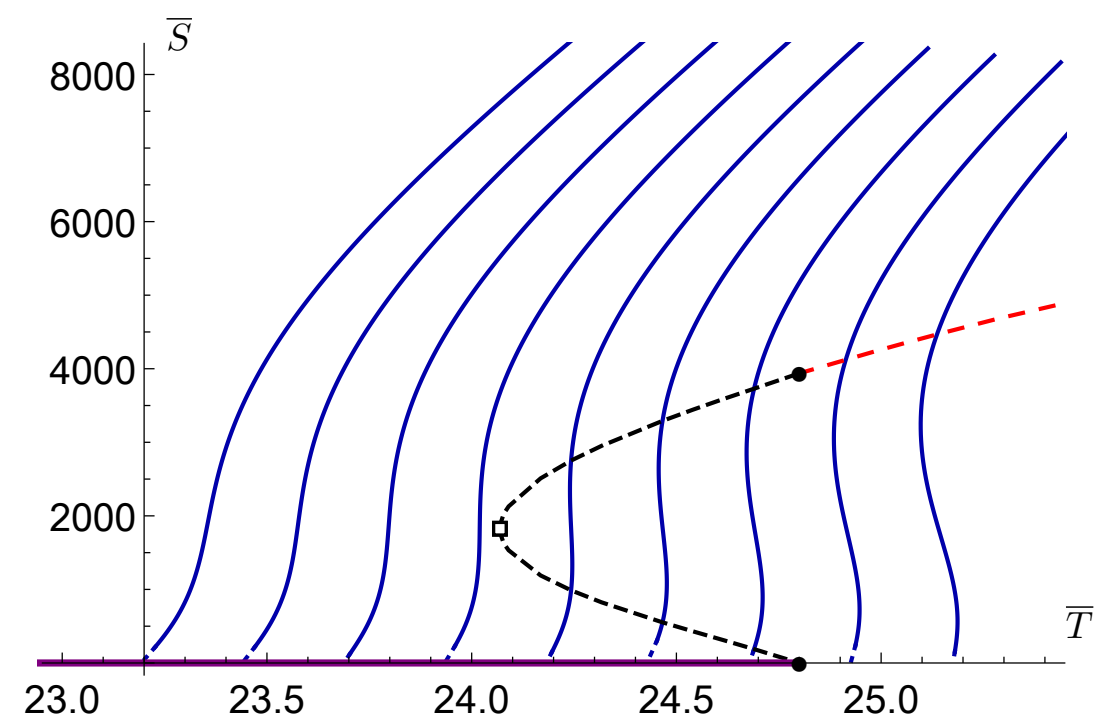

Figure 9. Entropy density for different theories with $b_{0}$ varying from $b_{0}=0.6783 \lesssim b_{0}^{\text {critical }}$ to $b_{0}=0.6861 \gtrsim b_{0}^{\text {triple }}$ from left to right, with an approximate separation of $\Delta b_{0} \approx 0.001$ between adjacent curves. The dashed, red curve indicates first-order phase transitions between a black brane phase and a regular horizonless phase with a discontinuous jump in the entropy density. The dashed, black curve indicates first-order phase transitions between two black branes. The turning point of this line, indicated with a square, corresponds to the second-order phase transition at the critical point. The solid, purple line on the axis indicates first-order transitions between the zero-entropy limit of a black brane branch and a regular horizonless solution. The entropy density is continuous across these transitions. The phase transition corresponding to the triple point is indicated with two black dots. The region close to the horizontal axis of the blue curves, shown with dashes, is the result of an extrapolation to zero entropy.
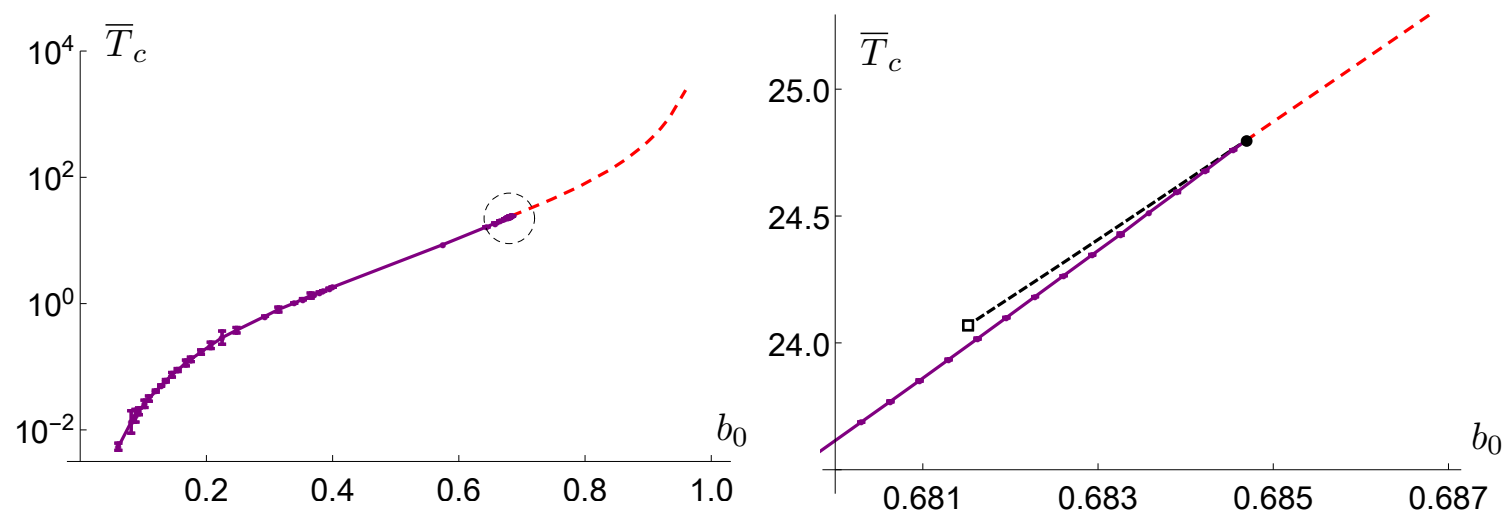

Figure 10. Phase diagram for the entire family of gauge theories (left) and zoomed in version of the circle around $b_{0} \approx 0.68$. The dashed, red line represents first-order transitions between a black brane and a regular horizonless solution with a discontinuity in the entropy density (Case A). The dashed, black line corresponds to phase transitions between two black brane solutions (Case B). The dotted, purple line indicates first-order transitions between the zero-entropy limit of a black brane branch and a regular horizonless solution. The entropy density is continuous across these transitions (Cases B and C). The purple curve requires an extrapolation to zero entropy. We have estimated the associated error bars, shown explicitly in the figure, by comparing a linear and a quadratic extrapolation. 


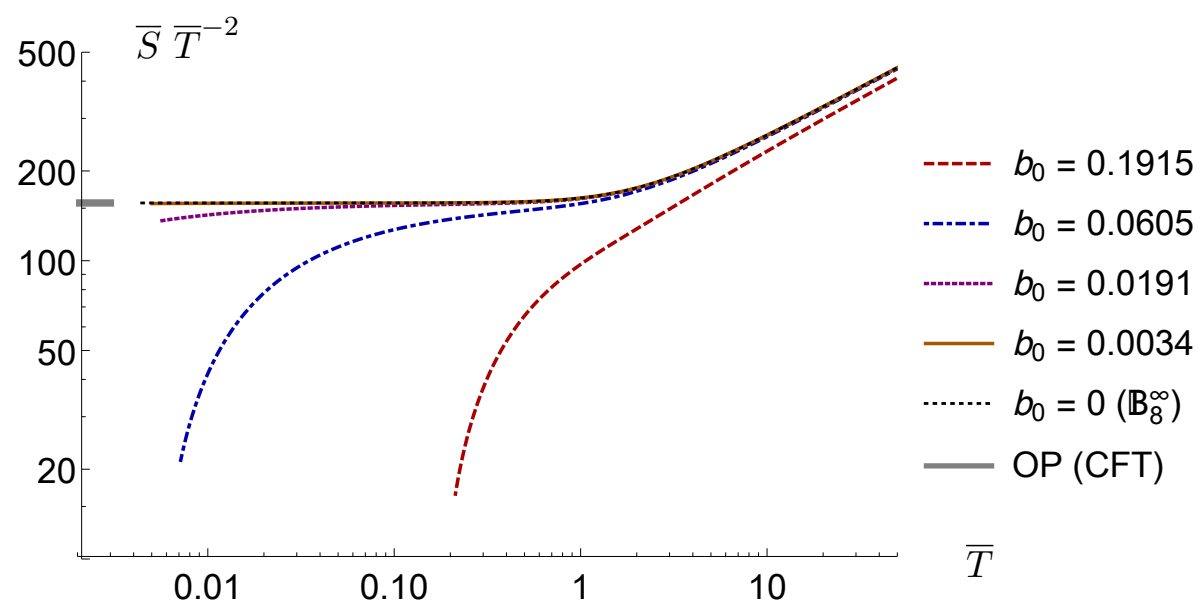

Figure 11. Dimensionless combination $S T^{-2}$ for different flows with $b_{0} \gtrsim 0$. This quantity is constant in a CFT at finite temperature, as in the IR of $\mathbb{B}_{8}^{\infty}$.

information we can draw the phase diagram of the entire family in the $\left(b_{0}, T\right)$-plane, which is shown in figure 10. The region above the curves of critical temperatures in the left panel is dominated by black branes and corresponds to ungapped phases in the dual gauge theories. Below these curves, the regular horizonless solutions are dominant and the preferred phases are gapped. The right panel zooms into the range of values that include Case B, where two transitions take place. The triple point, where the three lines meet, is indicated with a black dot. The critical point, where the line of first-order phase transitions between black branes ends, is indicated with a square.

\subsection{Quasi-conformal thermodynamics}

As described in section 2, there are two special values of the parameter with distinct IR dynamics. When $b_{0}=0$ the ground state $R G$ flow, denoted by $\mathbb{B}_{8}^{\infty}$, ends on a fixed point, as indicated by the leftmost arrow in figure 1 . The properties of this particular theory at finite temperature were already discussed around eq. (3.12). In this section we comment on the imprints that this fixed point leaves on the thermodynamics of flows passing nearby.

Conformal invariance dictates that when a three-dimensional CFT is heated up, the entropy grows as $S \propto T^{2}$. Flows with $b_{0} \gtrsim 0$ approach the fixed point but eventually fail to reach it, so it is expected that the entropy should scale approximately as in a CFT for some range of temperatures. This behaviour is confirmed by the results plotted in figure 11. For $\mathbb{B}_{8}^{\infty}$, the dimensionless quantity $S T^{-2}$ becomes nearly independent of $T$ for all temperatures below eq. (3.13). For black brane solutions with a small but non-zero value of $b_{0}$, a plateau develops before their zero-entropy limit is eventually reached at a finite temperature. The size and the steepness of the plateau, in which there is quasi-conformal behaviour, is controlled by the smallness of $b_{0}$.

\subsection{Quasi-confining thermodynamics}

For $b_{0}=1$ the solution is $\mathbb{B}_{8}^{\text {conf }}$, at the right of figure 1 . This theory is not only gapped but truly confining, in the sense that the quark-antiquark potential displays an area law 

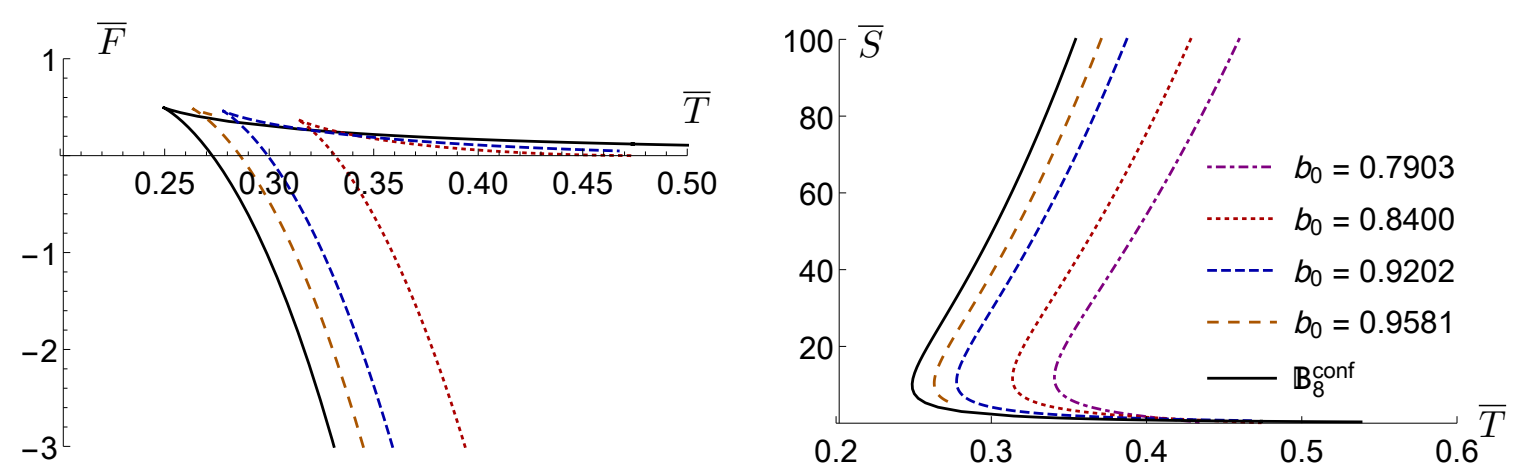

Figure 12. Free energy (left) and entropy (right) for different $\mathbb{B}_{8}$ metrics with $b_{0} \lesssim 1$, with the appropriate scaling of the charges, in dashed and dotted lines. In the limit $b_{0} \rightarrow 1$, we recover those of $\mathbb{B}_{8}^{\text {conf }}$, shown in solid black line.

(see also the arguments in [20]). In [10] this particularity was attributed to the vanishing of the CS level, $k=0$. Due to this, if we aim to understand the solution as a limit of the $\mathbb{B}_{8}$ family of metrics, we have to rescale the charge $Q_{k}$ - related to the CS level by eq. (3.2) - before taking $k \rightarrow 0$ (see [10, 29] for further details). Moreover, the radial coordinate in eq. (3.5) is no longer valid, so we define a new one through

$$
\mathrm{d} r=-\frac{\rho_{0}}{\xi^{2} \sqrt{1-\xi^{4}}} \mathrm{~d} \xi, \quad \xi \in(0,1)
$$

with $\rho_{0}$ a constant with dimensions of length. We factor out the charges from the fluxes, eq. (A.12), to obtain dimensionless functions as

$$
b_{J}=\frac{Q_{c}}{4 q_{c}}+\frac{2 q_{c}}{3 \rho_{0}} \mathcal{B}_{J}, \quad b_{X}=-\frac{Q_{c}}{4 q_{c}}-\frac{2 q_{c}}{3 \rho_{0}} \mathcal{B}_{X}, \quad a_{J}=-\frac{q_{c}}{2}-q_{c} \mathcal{A}_{J}, \quad h=\frac{128 q_{c}^{2}}{9 \rho_{0}^{6}} \mathbf{h} .
$$

The free parameters in the expansions both at the UV and at the horizon are essentially the same as in the rest of the $\mathbb{B}_{8}$ family, since $\xi$ and $u$ coincide asymptotically. However, now we need to gauge fix $f_{1}=0$ to compare with the ground state solution. After the appropriate rescaling in order to compare the same dimensionless quantities, it can be seen that the free energies and entropies for different values of $b_{0}$ approach those of $\mathbb{B}_{8}^{\text {conf }}$ as $b_{0} \rightarrow 1$. This is shown in figure 12. In the case of $\mathbb{B}_{8}^{\text {conf }}$, as we remove the horizon, that is, in the limit $S \rightarrow 0$, the temperature diverges, as in the case of small black branes in global AdS spacetimes. This branch is however unstable: before reaching it, there is a first-order phase transition at a critical temperature $\bar{T}_{c} \approx 0.27$. In the dual gauge theory this corresponds to a genuine confinement/deconfinement first-order phase transition.

\subsection{Regime of validity}

In this section we determine the range of validity of the supergravity solutions we have studied. For the ground state, as well as for the low temperature solutions considered in section 2, there are two requisites, already discussed in [10]. In this reference it was shown that the type IIA picture is trustable for energies in the gauge theory

$$
U \ll \lambda\left(1+\frac{\bar{M}^{2}}{2 N|k|}\right)\left(1-b_{0}^{2}\right),
$$


above which the perturbative regime is adequate. ${ }^{2}$ On the other hand, the IR is regular in eleven dimensions. In order for the curvature to be small it is required that

$$
\frac{\bar{M}^{2}}{2}+N|k| \gg 1
$$

On top of this, we have seen for instance in figure 3 that the curvature at the horizon grows unbounded as we remove it. This is important for phase transitions in Cases B and $\mathrm{C}$, since they occur precisely in this limit. As we approach the critical temperature, the eleven-dimensional Ricci scalar in Planck units grows as

$$
\ell_{p}^{2} R \sim\left(\frac{\bar{M}^{2}}{2}+N|k|\right)^{-1 / 3}\left(\bar{T}-\bar{T}_{c}\right)^{-2 / 3} .
$$

Therefore, there is always an interval of temperatures

$$
\bar{T} \in\left(\bar{T}_{c}, \bar{T}_{c}+\left(\frac{\bar{M}^{2}}{2}+N|k|\right)^{-1 / 2}\right),
$$

where curvatures are large and higher-order curvature corrections must be considered. Nevertheless, this region is small provided eq. (4.10) is satisfied.

\section{Conclusions and discussion}

In this work we have studied the finite-temperature physics of a family of gauge theories in three dimensions by means of holography. The UV, which is weakly coupled, is expected to be described by a two-sites quiver Yang-Mills theory deformed by CS-terms, as discussed around eq. (2.3). The parameter $b_{0}$, labelling the different solutions within the family, controls the asymptotic difference between the microscopic couplings of each of the two factors in the quiver. The IR of the ground state is generically gapped except for a particular value of the parameter that drives the RG flow to a fixed point. Despite the gap, the models do not exhibit a linear quark-antiquark potential at large separations unless the CS level is zero [10].

The gap is lost at some non-zero critical temperature, as we have shown by constructing the black brane geometries dual to the ungapped phase and demonstrating their dominance above $T_{c}$. The nature of the phase transition depends on the value of $b_{0}$ and there are three distinct regions. For large values of the parameter there is a single degapping first-order transition. At intermediate values, there is a small range in which two transitions take place: as the temperature is decreased from high values, there is a first-order transition between two different black branes, corresponding to two ungapped states, while at a lower critical temperature there is a degapping first-order transition at which the entropy is continuous. Finally, at even lower values of the parameter there is only a phase transition of this second type. This is summarised in the phase diagram of figure 10, where a triple point and a critical point are indicated by a black dot and by a square, respectively.

\footnotetext{
${ }^{2}$ Recall that the three-dimensional gauge theories are superrenormalizable and asymptotically free.
} 

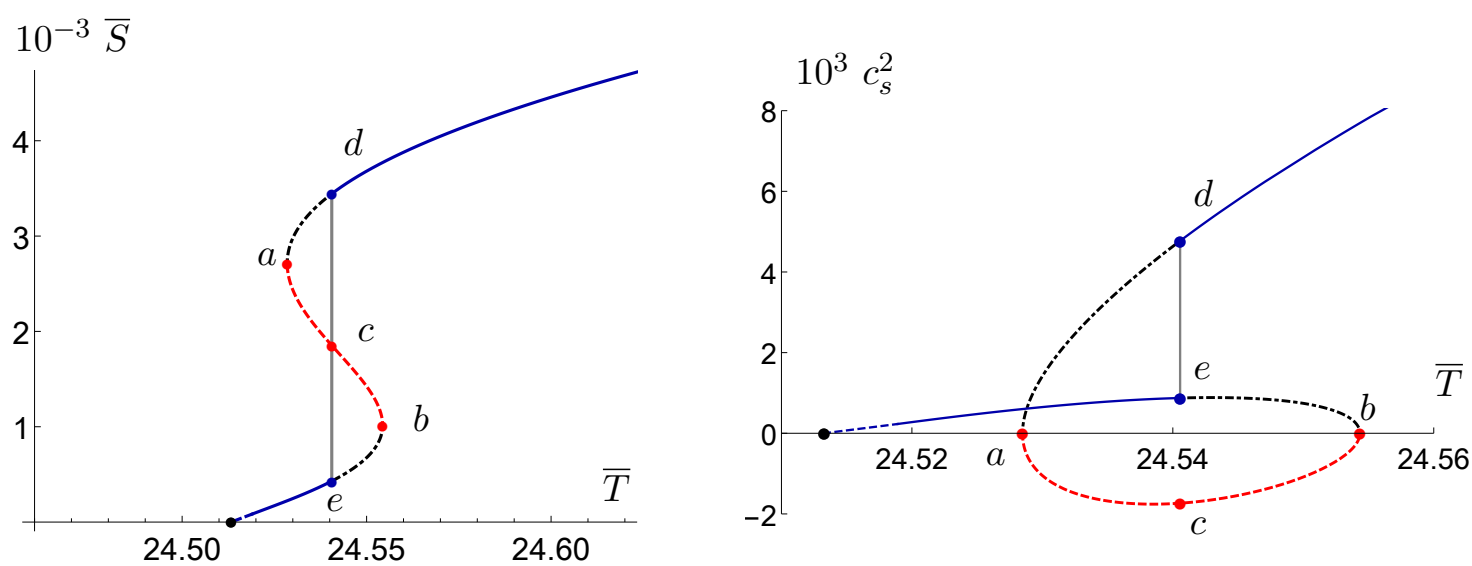

Figure 13. Entropy density (left) and speed of sound squared (right) as a function of temperature for black branes in the theory with $b_{0}=0.6835$ (Case B).

At the critical point the phase transition is of course second-order. An analogous situation was found in [22], where (in that case a bottom-up) holographic model with a critical point was studied. As expected on general grounds, the order parameter at the second-order phase transition can be constructed out of physical observables that jump discontinuously across the line of first-order phase transitions and whose susceptibilities diverge at the critical point. As in [22], in our case this will be a combination of the energy density $E$ and the VEVs of (some) scalar operators dual to the scalar fields in the bulk. The specific combination could be determined via a calculation on the gravity side. In this description fluctuations of the metric mix with those of the scalar fields and the mode whose correlation length diverges at the critical point will be a linear combination of these fluctuations.

In figure 13(left) we reproduce again the entropy density of figure 6 for Case B, which is the richest. A universal feature of a first-order phase transition is that the so-called "spinodal region" between points $a$ and $b$ is locally thermodynamically unstable, since the specific heat given in the first equation in (4.5) is negative, $c_{v}<0$. It follows from the second equation in (4.5) that this region is also locally dynamically unstable, since it also has negative speed of sound squared. This is confirmed by the plot in figure 13(right). We see that, as expected, the speed of sound squared crosses zero at the points $a$ and $b$ but, interestingly, also at the zero-entropy point where the transition to the confined phase takes place. For detailed discussions of the spinodal instability and the associated dynamics in the holographic context see e.g. [23-27].

As noted below eq. (4.6), the size of the intermediate region where two phase transitions take place is very small compared to the range of the $b_{0}$ parameter. In other words, $b_{0}^{\text {triple }}-b_{0}^{\text {critical }}=0.0032 \ll 1$. Nevertheless, we have verified that the existence of this intermediate region is not a numerical artifact by integrating the equations with two different integrators and by varying the control parameters in each of them. It would be interesting to understand in detail how such a small region arises dynamically in our model. A dynamically-generated small parameter was also encountered in [30] in the phase diagram of a holographic color superconductor. 


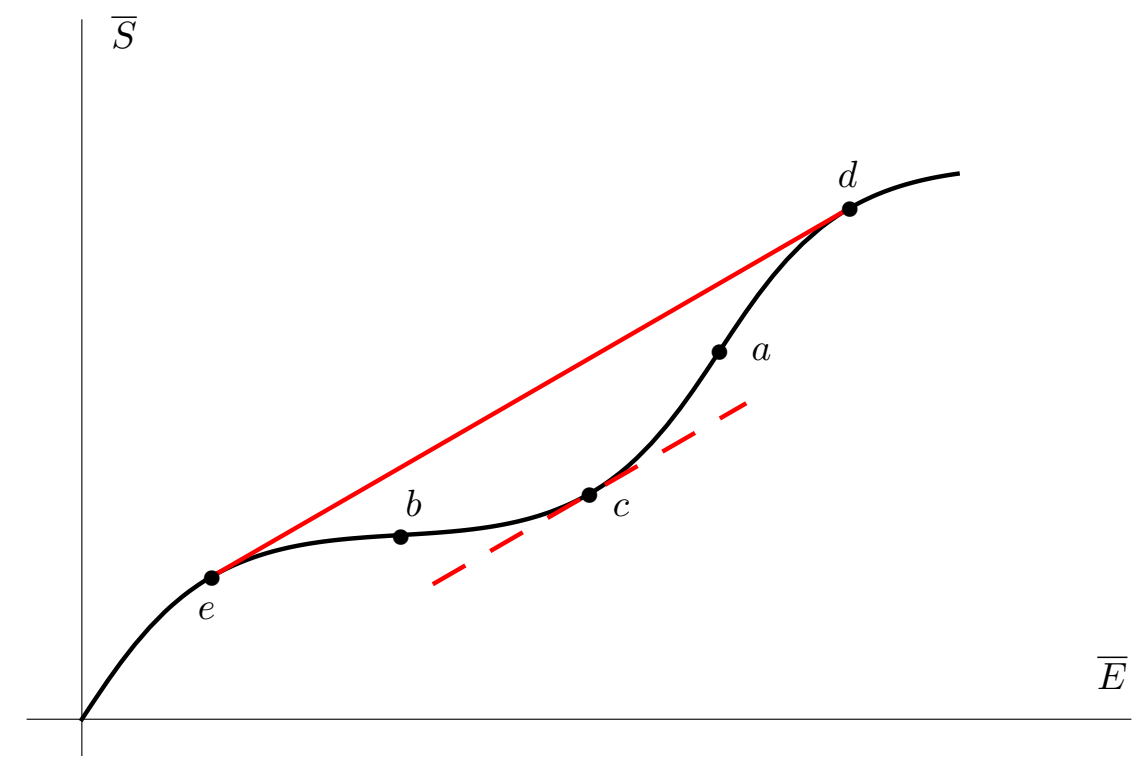

Figure 14. Qualitative form of the entropy density in the microcanonical ensemble. The solid red segment corresponds to the average entropy density of phase-separated configurations, as explained in the text. The dashed red segment indicates that the slope of the tangent at point $c$ is the same as that of the solid red segment.

In this paper we have focused on the behaviour of the system in the canonical ensemble, in which the control parameter is the temperature and the preferred state is the one that minimises the free energy. However, the behaviour in the microcanonical ensemble in the infinite-volume limit that we work in follows from general arguments (see [27] for a discussion at finite volume in the holographic context). Consider again Case B, since it is the richest. In the microcanonical ensemble the control parameter is the energy and the preferred state is the one that maximises the entropy. The qualitative form of the function $S(E)$ is shown in figure 14. The key features are as follows. $S$ is convex $\left(S^{\prime \prime}(E)>0\right)$ in the region between $a$ and $b$. This indicates local thermodynamical instability, since the system can increase its total entropy by rising the energy slightly in part of its volume and lowering in another so as to keep the total energy fixed. In the regions $e-b$ and $a-d$ the entropy function is concave $\left(S^{\prime \prime}(E)<0\right)$ but there are states with the same total energy and higher total entropy, namely phase-separated configurations in which the phases $e$ and $d$ coexist at the critical temperature. These states are characterised by the fractions $0 \leq x,(1-x) \leq 1$ of the total volume occupied by each phase, so their total entropy is of the form $S_{e}+\left(S_{d}-S_{e}\right) x$, as indicated by the red segment in figure 14 . Therefore the regions $e-b$ and $a-d$ are locally but not globally thermodynamically stable. Finally, all states outside the region $e-d$ are globally stable. For our system, these qualitative features are difficult to appreciate directly on a plot of $S$ versus $E$ because the curve $S(E)$ is very close to a straight line. For this reason we show the convexity/concavity property (the second derivative) in figure 15(left) and the difference between the phase-separated configurations and the homogeneous solutions in figure 15(right). 

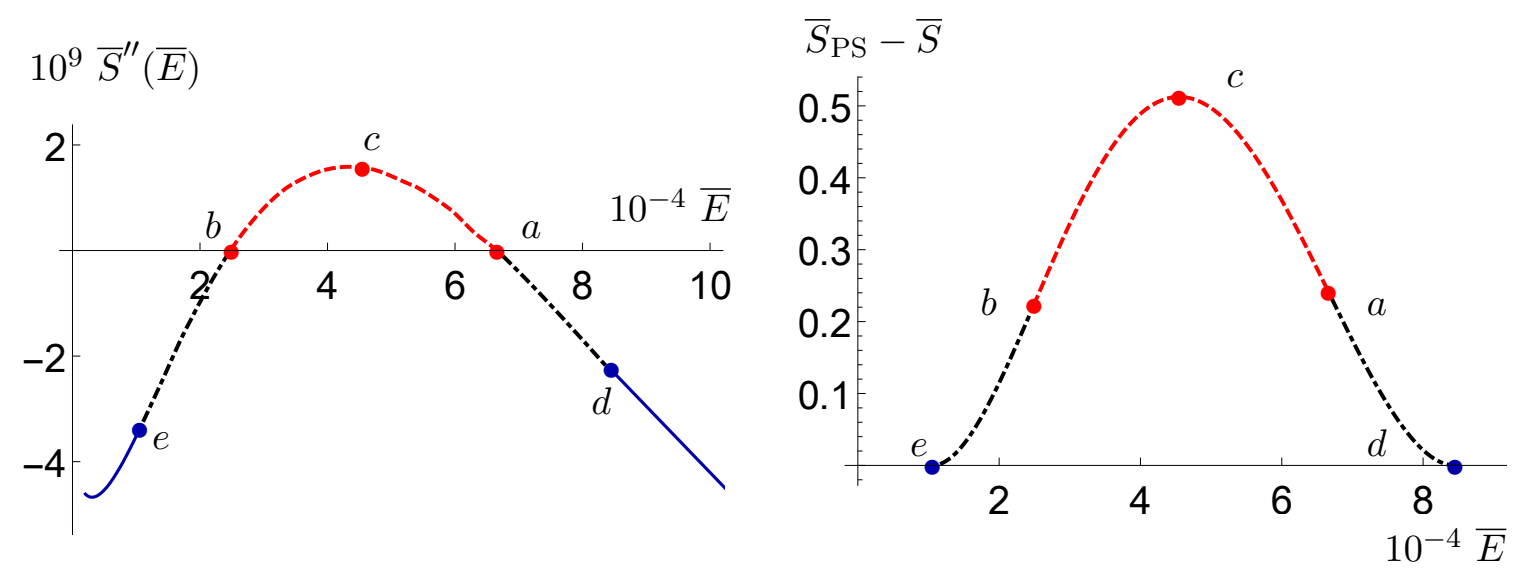

Figure 15. (Left) Second derivative $S^{\prime \prime}(E)$ of the entropy density with respect to the energy density, showing the convexity/concavity properties discussed in the text. (Right) Difference between the average entropy density of the phase-separated configurations and the entropy density of the homogeneous solutions, showing that the former are preferred in the region between $a$ and $b$. As in figure 13 these plots correspond to the theory with $b_{0}=0.6835$ (Case B).

The black branes that we have found reach a finite temperature in the limit in which the horizon is removed and the entropy vanishes. As a result, the transition for low values of $b_{0}$ seems to take place at zero entropy but finite temperature. However, the geometries recovered in this limit, which were identified in section 3.3, are singular. This means that, for temperatures slightly above the critical one, the curvature is large and the supergravity approximation is unreliable in a small region near the horizon, as detailed in section 4.5. The singularity is a good one according to the classification of [28], since by construction it can be cloaked behind a horizon. It would be interesting to discern how string theory resolves the singularity and how the phase transition manifests itself in that picture.

An analogous phase transition was observed in [31], where black branes in a similar system were obtained. In that case the ground state is conformal in the UV and preserves $\mathcal{N}=2$, being a higher-supersymmetric version of the $R G$ flow denoted $\mathbb{B}_{8}^{\text {OP }}$ at the left of figure 1. Our results can also be compared to those in [15, 16], where the Klebanov-Tseytlin and Klebanov-Strassler theories at finite temperature were studied. The phase transition shown to happen in those models is comparable to the one encountered here for $b_{0}=1$. Indeed, when the CS-level vanishes, which happens for the flow called $\mathbb{B}_{8}^{\text {conf }}$ at the right of figure $1,{ }^{3}$ the first-order phase transition is genuinely a confinement/deconfinement transition. Yet, the UV of our models, which are asymptotically free, is simpler than the infinite cascade of their four-dimensional cousins in $[15,16]$. It would also be interesting to compare to the phase structure of the $\mathcal{N}=1^{*}$ super Yang-Mills theory, the high temperature deconfined phase of which was studied in [33].

We have computed the different Maxwell charges of our black brane solutions and verified that they do not change sign along the RG flows up to the horizon. In other words,

\footnotetext{
${ }^{3} \mathrm{~A}$ black brane solution in this background, perturbative in the number of fractional branes, was constructed in [32].
} 
the charges hidden behind the horizon have the same sign as those measured at infinity. This is expected [34-37] since our solutions are smeared along the internal directions of the geometry, as in [15, 31]. Some arguments (see e.g. [38-40] for recent analyses) suggest that a change of sign in the charges may be realised in solutions with localised horizons along the internal directions, whose existence may lend support to KKLT-type constructions of de Sitter vacua [41]. We leave the investigation of these aspects for the future.

\section{Acknowledgments}

We thank David Pravos for collaboration in an early stage of the project. We were supported by grants FPA2016-76005-C2-1-P, FPA2016-76005-C2-2-P, SGR-2017-754, MDM2014-0369 of ICCUB, and ERC Starting Grant HoloLHC-306605. JGS acknowledges support from the FPU program, fellowship FPU15/02551. DE was supported by the OCEVU Labex (ANR-11-LABX-0060) and the A*MIDEX project (ANR-11-IDEX-0001-02) funded by the "Investissements d'Aveni" French government program managed by the ANR. AF is supported by the "Beatriz Galindo" program, as well as grant PGC2018-096894-B-100.

\section{A Ansatz and equations of motion}

In this appendix we give the details needed to reproduce the equations of motion that we solve in the bulk of the paper, both from the ten- and four-dimensional points of view.

\section{A.1 Ten-dimensional ansatz}

Although the ground state solutions are regular only in eleven dimensions, we will give the details of the ten-dimensional setup, since the geometric picture is clearer. In string frame, the equations of motion for the forms of type IIA supergravity read

$$
\begin{aligned}
\mathrm{d} * F_{4}+H & \wedge F_{4}=0, \\
\mathrm{~d} * F_{2}+H \wedge * F_{4} & =0, \\
\mathrm{~d}\left(e^{-2 \Phi} * H\right)-F_{2} \wedge * F_{4}-\frac{1}{2} F_{4} \wedge F_{4} & =0 .
\end{aligned}
$$

The field strengths are such that the following Bianchi identities must be satisfied

$$
\mathrm{d} H=0, \quad \mathrm{~d} F_{2}=0, \quad \mathrm{~d} F_{4}=H \wedge F_{2} .
$$

On top of that, we have the equations governing the dilaton

$$
R+4 \nabla_{M} \nabla^{M} \Phi-4 \nabla^{M} \Phi \nabla_{M} \Phi-\frac{1}{12} H^{2}=0,
$$

and the metric

$$
R_{M N}+2 \nabla_{M} \nabla_{N} \Phi-\frac{1}{4} H_{M N}^{2}=e^{2 \Phi}\left[\frac{1}{2}\left(F_{2}^{2}\right)_{M N}+\frac{1}{12}\left(F_{4}^{2}\right)_{M N}-\frac{1}{4} g_{M N}\left(\frac{1}{2} F_{2}^{2}+\frac{1}{24} F_{4}^{2}\right)\right],
$$

in a self-explanatory notation. 
All the solutions considered are based on the $\mathbb{C P}^{3}$ as internal manifold and asymptote to a stack of coincident D2-branes in the UV. We then take as ansatz for the metric and dilaton

$$
\begin{aligned}
\mathrm{d} s_{\mathrm{st}}^{2} & =h^{-\frac{1}{2}}\left(-\mathrm{b} \mathrm{d} t^{2}+\mathrm{d} x_{1}^{2}+\mathrm{d} x_{2}^{2}\right)+h^{\frac{1}{2}}\left(\frac{\mathrm{d} r^{2}}{\mathrm{~b}}+e^{2 f} \mathrm{~d} \Omega_{4}^{2}+e^{2 g}\left[\left(E^{1}\right)^{2}+\left(E^{2}\right)^{2}\right]\right) \\
e^{\Phi} & =h^{\frac{1}{4}} e^{\Lambda}
\end{aligned}
$$

where the functions $f, g, h, \mathrm{~b}$ and $\Lambda$ all depend on the radial coordinate. In this picture the complex projective plane is seen as the coset $\mathrm{Sp}(2) / \mathrm{U}(2)$. This is a $\mathrm{S}^{2}$, described by the vielbeins $E^{1}$ and $E^{2}$, fibered over $\mathrm{S}^{4}$, with metric $\mathrm{d} \Omega_{4}^{2}$. A suitable choice of coordinates is the following. Let $\omega^{i}$ be a set of left invariant forms on the three-sphere. Then the metric of the unit-radius four-sphere can be written as

$$
\mathrm{d} \Omega_{4}^{2}=\frac{4}{\left(1+\xi^{2}\right)^{2}}\left[\mathrm{~d} \xi^{2}+\frac{\xi^{2}}{4} \omega^{i} \omega^{i}\right]
$$

with $\xi$ a non-compact coordinate. If we parametrise the two-sphere by the angles $\theta$ and $\varphi$, then the non-trivial fibration is described by the vielbeins

$$
\begin{aligned}
& E^{1}=\mathrm{d} \theta+\frac{\xi^{2}}{1+\xi^{2}}\left(\sin \varphi \omega^{1}-\cos \varphi \omega^{2}\right) \\
& E^{2}=\sin \theta\left(\mathrm{d} \varphi-\frac{\xi^{2}}{1+\xi^{2}} \omega^{3}\right)+\frac{\xi^{2}}{1+\xi^{2}} \cos \theta\left(\cos \varphi \omega^{1}+\sin \varphi \omega^{2}\right) .
\end{aligned}
$$

For our purposes, it is convenient to consider a rotated version of the vielbeins on the four-sphere that read:

$$
\begin{aligned}
\mathcal{S}^{1} & =\frac{\xi}{1+\xi^{2}}\left[\sin \varphi \omega^{1}-\cos \varphi \omega^{2}\right], \\
\mathcal{S}^{2} & =\frac{\xi}{1+\xi^{2}}\left[\sin \theta \omega^{3}-\cos \theta\left(\cos \varphi \omega^{1}+\sin \varphi \omega^{2}\right)\right], \\
\mathcal{S}^{3} & =\frac{\xi}{1+\xi^{2}}\left[\cos \theta \omega^{3}+\sin \theta\left(\cos \varphi \omega^{1}+\sin \varphi \omega^{2}\right)\right], \\
\mathcal{S}^{4} & =\frac{2}{1+\xi^{2}} \mathrm{~d} \xi .
\end{aligned}
$$

Despite their explicit dependence on the angles of the two-sphere, it can be easily checked that $\mathcal{S}^{n} \mathcal{S}^{n}=\mathrm{d} \Omega_{4}^{2}$. Using these vielbeins we can construct a set of left-invariant forms on the coset. This set contains the two-forms

$$
X_{2}=E^{1} \wedge E^{2}, \quad J_{2}=\mathcal{S}^{1} \wedge \mathcal{S}^{2}+\mathcal{S}^{3} \wedge \mathcal{S}^{4},
$$

as well as the three-forms

$$
\begin{aligned}
X_{3} & =E^{1} \wedge\left(\mathcal{S}^{1} \wedge \mathcal{S}^{3}-\mathcal{S}^{2} \wedge \mathcal{S}^{4}\right)-E^{2} \wedge\left(\mathcal{S}^{1} \wedge \mathcal{S}^{4}+\mathcal{S}^{2} \wedge \mathcal{S}^{3}\right) \\
J_{3} & =-E^{1} \wedge\left(\mathcal{S}^{1} \wedge \mathcal{S}^{4}+\mathcal{S}^{2} \wedge \mathcal{S}^{3}\right)-E^{2} \wedge\left(\mathcal{S}^{1} \wedge \mathcal{S}^{3}-\mathcal{S}^{2} \wedge \mathcal{S}^{4}\right)
\end{aligned}
$$


These are related by exterior differentiation as

$$
\mathrm{d} X_{2}=\mathrm{d} J_{2}=X_{3}, \quad \mathrm{~d} J_{3}=2\left(X_{2} \wedge J_{2}+J_{2} \wedge J_{2}\right) .
$$

Higher forms constructed by wedging of these are also left invariant, so we have the two four-forms $X_{2} \wedge J_{2}$ and $J_{2} \wedge J_{2}$ appearing in the equation above together with the volume form $\Omega_{6}=-\left(E_{1} \wedge E_{2}\right) \wedge\left(\mathcal{S}^{1} \wedge \mathcal{S}^{2} \wedge \mathcal{S}^{3} \wedge \mathcal{S}^{4}\right)$. There are no adequate one- or five-forms. We will write the fluxes in terms of these left-invariant forms, since this symmetry ensures the consistency of the ansatz, meaning that all the internal angles will drop from the resulting equations, which will depend just on the radial coordinate. Thus, we take the following forms

$$
\begin{aligned}
& F_{4}=\mathbf{f}_{4} * \Omega_{6}+G_{4}+B_{2} \wedge F_{2}, \quad F_{2}=Q_{k}\left(X_{2}-J_{2}\right), \\
& G_{4}=\mathrm{d}\left(a_{J} J_{3}\right)+q_{c}\left(J_{2} \wedge J_{2}-X_{2} \wedge J_{2}\right), \quad B_{2}=b_{X} X_{2}+b_{J} J_{2},
\end{aligned}
$$

where we have defined the quantity

$$
\mathbf{f}_{4}=Q_{k} b_{J}^{2}+2\left(q_{c}+2 a_{J}\right) b_{X}-2 b_{J}\left(q_{c}-2 a_{J}+Q_{k} b_{X}\right)+Q_{c} .
$$

The parameters $Q_{c}, q_{c}$ and $Q_{k}$ are constants, related to gauge theory parameters as stated around eq. (3.2). On the other hand, $b_{J}, b_{X}$ and $a_{J}$ depend on the radial coordinate, their dynamics dictated by the form equations (A.1).

The ground state is supersymmetric and has $b=1$. The explicit form of the rest of the functions is not important for our purposes and can be found in [10]. Finally, in eleven dimensions the backgrounds correspond to M2-branes

$$
\mathrm{d} s_{11}^{2}=H^{-2 / 3} \mathrm{~d} x_{1,2}^{2}+H^{1 / 3} \mathrm{~d} s_{8}^{2},
$$

with eight-dimensional transverse space

$$
\mathrm{d} s_{8}^{2}=e^{-\Lambda}\left[\mathrm{d} r^{2}+e^{2 f} \mathrm{~d} \Omega_{4}^{2}+e^{2 g}\left[\left(E^{1}\right)^{2}+\left(E^{2}\right)^{2}\right]\right]+e^{\Lambda} Q_{k}^{2}\left(\mathrm{~d} \psi-\cos \theta \mathrm{d} \varphi+\xi \mathcal{S}^{3}\right)^{2}
$$

\section{A.2 Four-dimensional reduction}

To study aspects like spectra [29] or thermodynamic properties it is convenient to work with a four-dimensional consistent truncation. This truncation was presented in [10]. In order to reduce from ten to four dimensions, we take the following metric

$$
\mathrm{d} s_{\mathrm{st}}^{2}=e^{\Phi / 2}\left(e^{-2 U-4 V} \mathrm{~d} s_{4}^{2}+e^{2 U} \frac{1}{4}\left[\left(E^{1}\right)^{2}+\left(E^{2}\right)^{2}\right]+e^{2 V} \frac{1}{2} \mathrm{~d} \Omega_{4}^{2}\right) .
$$

The forms read exactly as in eq. (A.12). Assuming that all the functions depend just on the coordinates in $\mathrm{d} s_{4}^{2}$, the resulting equations of motion can be obtained from the action

$$
\begin{aligned}
S_{4}=\frac{1}{2 \kappa_{4}^{2}} \int\left[R * 1+\mathcal{L}_{\text {kin }}-\mathcal{V} * 1\right] \\
=\frac{1}{2 \kappa_{4}^{2}} \int\left[R * 1-\frac{1}{2}(\mathrm{~d} \Phi)^{2}-4(\mathrm{~d} U)^{2}-12(\mathrm{~d} V)^{2}-8 \mathrm{~d} U \cdot \mathrm{d} V-4 e^{-4 V-\Phi}\left(\mathrm{d} b_{J}\right)^{2}\right. \\
\left.\quad-8 e^{-4 U-\Phi}\left(\mathrm{d} b_{X}\right)^{2}-32 e^{-2 U-4 V+\Phi / 2}\left(\mathrm{~d} a_{J}\right)^{2}-\mathcal{V} * 1\right]
\end{aligned}
$$


with the potential

$$
\begin{aligned}
\mathcal{V}= & 128 e^{-6 U-12 V-\Phi / 2}\left[Q_{c}+4 a_{J}\left(b_{J}+b_{X}\right)+Q_{k} b_{J}\left(b_{J}-2 b_{X}\right)+2 q_{c}\left(b_{X}-b_{J}\right)\right]^{2} \\
& +32\left(b_{J}+b_{X}\right)^{2} e^{-4 U-8 V-\Phi}+64\left[2 a_{J}+Q_{k}\left(b_{J}-b_{X}\right)-q_{c}\right]^{2} e^{-6 U-8 V+\Phi / 2} \\
& +32\left(2 a_{J}-Q_{k} b_{J}+q_{c}\right)^{2} e^{-2 U-12 V+\Phi / 2}+4 Q_{k}^{2} e^{-2 U-8 V+3 \Phi / 2} \\
& +8 Q_{k}^{2} e^{-6 U-4 V+3 \Phi / 2}-24 e^{-2 U-6 V}-8 e^{-4 U-4 V}+2 e^{-8 V}
\end{aligned}
$$

Thus, the action contains six scalars: $U$ and $V$ from the metric, $b_{X}, b_{J}$ and $a_{J}$ from the fluxes (RR and NS forms) plus the dilaton $\Phi$. By comparing eq. (A.16) with eq. (2.1) it is possible to translate into the variables used in the bulk of the paper. Additionally, we have three constants, $Q_{c}, Q_{k}$ and $q_{c}$, whose relation with gauge-theory parameters was reported in eq. (3.2). The potential can be recovered from the superpotential

$$
\begin{aligned}
\mathcal{W}= & e^{-4 V}+2 e^{-2 U-2 V}+Q_{k} e^{-3 U-2 V+3 \Phi / 4}-Q_{k} e^{-U-4 V+3 \Phi / 4} \\
& -4 e^{-3 U-6 V-\Phi / 4}\left[Q_{c}+4 a_{J}\left(b_{J}+b_{X}\right)+Q_{k} b_{J}\left(b_{J}-2 b_{X}\right)+2 q_{c}\left(b_{X}-b_{J}\right)\right]
\end{aligned}
$$

through the usual relation

$$
\mathcal{V}=4 G^{i j} \partial_{i} \mathcal{W} \partial_{j} \mathcal{W}-6 \mathcal{W}^{2}
$$

We now perform standard holographic renormalization on this action. As a first step, we cut off the radial coordinate at some $\mu_{\mathrm{Uv}}$. The action diverges in the limit $\mu_{\mathrm{UV}} \rightarrow \infty$, so we have to find the counterterms $S_{\text {ct }}$ that regularize it. If these are correctly chosen, adding them to the action and removing the regulator gives a finite quantity. Additionally, we have to include the Gibbons-Hawking term [42]. The regularized action is thus

$$
S_{\mathrm{reg}}=\frac{1}{2 \kappa_{4}^{2}} \int_{\mathcal{M}}\left[R * 1+\mathcal{L}_{\mathrm{kin}}-\mathcal{V} * 1\right]+S_{\mathrm{GH}}+S_{\mathrm{ct}},
$$

where $\mathcal{M}$ is the whole four dimensional spacetime, $S_{\mathrm{ct}}$ is the counterterm piece and $S_{\mathrm{GH}}$ is the Gibbons-Hawking term

$$
S_{\mathrm{GH}}=\frac{1}{\kappa_{4}^{2}} \int_{\partial \mathcal{M}} K * 1,
$$

being $K$ the extrinsic curvature induced on the boundary $\partial \mathcal{M}$.

Introducing a horizon in the gravitational description and thus considering the gauge theory at finite temperature is an infrared deformation. On the other hand, it is known that the superpotential renormalizes the supersymmetric solution. If the black brane enjoys the same UV asymptotic behaviour as the ground state, the UV divergences will be cancelled out in the same way. Therefore, the counterterm we consider is

$$
S_{\mathrm{ct}}=-\frac{2}{\kappa_{4}^{2}} \int_{\partial \mathcal{M}} \mathcal{W} * 1
$$

where $\mathcal{W}$ is the superpotential, eq. (A.19). The renormalized on-shell action used to extract the thermodynamic quantities is obtained through the usual manipulations as

$$
S_{\text {ren }}=\lim _{\mu_{\mathrm{UV}} \rightarrow \infty} S_{\text {reg }}=-\frac{\beta V_{2}}{2 \kappa_{4}^{2}} \lim _{\mu_{\mathrm{UV}} \rightarrow \infty}\left[\left.\sqrt{\gamma}\left(2 K^{t}{ }_{t}-2 K+4 \mathcal{W}\right)\right|_{\mu_{\mathrm{UV}}}-\left.2 \sqrt{\gamma} K^{t}{ }_{t}\right|_{H}\right]
$$


where the last term is to be evaluated at the horizon. Here $\gamma$ is the determinant of the boundary metric, $\beta$ is the period of the Euclidean time and $V_{2}$ is the volume of $\mathbb{R}^{2}$. From this renormalized action we can compute the energy-momentum tensor of the dual gauge theory by varying with respect to the induced metric, evaluated at the boundary

$$
T^{i}{ }_{j}=-\frac{1}{\kappa_{4}^{2}} \lim _{\mu_{\mathrm{UV}} \rightarrow \infty}\left\{\left.\sqrt{\gamma}\left(K^{i}{ }_{j}-\delta^{i}{ }_{j}(K-2 \mathcal{W})\right)\right|_{\mu_{\mathrm{UV}}}\right\}=\operatorname{diag}(-E, P, P)
$$

There is a final observation which is useful in the numerical computations. Given a four-dimensional metric of the form

$$
\mathrm{d} s_{4}^{2}=-g_{t t} \mathrm{~d} t^{2}+g_{x x} \mathrm{~d} x_{1}^{2}+g_{x x} \mathrm{~d} x_{2}^{2}+g_{r r} \mathrm{~d} r^{2}
$$

it can be seen that the action is invariant under

$$
g_{t t} \mapsto \mu^{2} g_{t t} \quad g_{x x} \mapsto \mu^{-1} g_{x x}
$$

Using Noether's theorem there must be a conserved current associated to this symmetry, which is

$$
j^{\mu}=\frac{g_{t t} g_{x x}^{\prime}-g_{t t}^{\prime} g_{x x}}{\sqrt{g_{t t} g_{r r}}} \delta_{r}^{\mu}
$$

satisfying

$$
\partial_{\mu} j^{\mu}=\partial_{r}\left(\frac{g_{t t} g_{x x}^{\prime}-g_{t t}^{\prime} g_{x x}}{\sqrt{g_{t t} g_{r r}}}\right)=0, \quad \Rightarrow \quad \frac{g_{t t} g_{x x}^{\prime}-g_{t t}^{\prime} g_{x x}}{\sqrt{g_{t t} g_{r r}}}=\text { constant }
$$

This equation relates a combination of UV parameters with horizon data as in eq. (3.9). This can be employed either as a check of the numerical coefficients obtained while solving the equations or, using it as an input, to reduce the number of unknown constants in the shooting problem.

\section{B UV expansions}

In this appendix we present some details of the UV expansions. The undetermined parameters are those in eq. (3.6), while the remaining ones, shown here, are given in terms of them. In the following expressions we have already fixed $f_{1}=-1$ as explained in the bulk of the paper. Although we only show a few terms, we indicate in the sums the order up to which we solved the equations - for example, up to order $u^{22}$ in the case of $e^{\mathcal{F}}$.

For the metric function $e^{\mathcal{F}}$ we expanded around $u=0$ as

$$
e^{\mathcal{F}}=\frac{1}{u \sqrt{2}}\left[1+\sum_{n=1}^{22} f_{n} u^{n}+\mathcal{O}\left(u^{23}\right)\right] .
$$


The first few coefficients read

$$
\begin{aligned}
& f_{2}=-\frac{5}{2}, \quad \\
& f_{6}=\frac{13}{120\left(b_{0}^{2}-1\right)}\left[3 b_{0}^{2}\left(52 \mathrm{~b}_{5}-12436 f_{4}+224 f_{5}-33671\right)-96\left(196 b_{4}-5 b_{6}\right) b_{0}\right. \\
& \left.+4 \mathrm{~b}_{5}-3\left(364 f_{4}+224 f_{5}+11705\right)\right], \\
& f_{7}=\frac{1}{2520\left(b_{0}^{2}-1\right)}\left[4\left(5321 b_{0}^{2}-141\right) \mathrm{b}_{5}-21\left(b_{0}^{2}\left(229456 f_{4}-3308 f_{5}+599069\right)\right.\right. \\
& \left.\left.+592\left(196 b_{4}-5 b_{6}\right) b_{0}+7344 f_{4}+3308 f_{5}+240387\right)\right] .
\end{aligned}
$$

Similarly, the function $e^{\mathcal{G}}$ enjoys an expansion

$$
e^{\mathcal{G}}=\frac{1}{2 u}\left[1+\sum_{n=1}^{22} g_{n} u^{n}+\mathcal{O}\left(u^{23}\right)\right],
$$

with the first coefficients being

$$
\begin{aligned}
g_{1}= & -2, \quad g_{2}=-4, \quad g_{3}=-8, \quad g_{4}=-2 f_{4}-\frac{205}{4}, \quad g_{5}=3 f_{4}+f_{5}+\frac{283}{4}, \\
g_{6}= & -\frac{b_{0}^{2}\left(-36 \mathrm{~b}_{5}+8160 f_{4}-72 f_{5}+22917\right)+21\left(196 b_{4}-5 b_{6}\right) b_{0}+\mathrm{b}_{5}+240 f_{4}+72 f_{5}+6861}{15\left(b_{0}^{2}-1\right)}, \\
g_{7}= & \frac{1}{315\left(b_{0}^{2}-1\right)}\left[\left(4624 b_{0}^{2}-354\right) \mathrm{b}_{5}\right. \\
& \left.-21\left(b_{0}^{2}\left(47780 f_{4}-308 f_{5}+141577\right)+122\left(196 b_{4}-5 b_{6}\right) b_{0}+1020 f_{4}+308 f_{5}+31419\right)\right] .
\end{aligned}
$$

Analogously, for the function $e^{\Lambda}$ we have

$$
e^{\Lambda}=1+\sum_{n=1}^{22} \lambda_{n} u^{n}+\mathcal{O}\left(u^{23}\right),
$$

where

$$
\begin{gathered}
\lambda_{1}=0, \quad \lambda_{2}=-4, \quad \lambda_{3}=-16, \quad \lambda_{4}=-48, \quad \lambda_{5}=\frac{6 f_{4}}{5}+2 f_{5}-\frac{71}{10}, \\
\lambda_{6}=\frac{2\left(b_{0}^{2}\left(5 \mathrm{~b}_{5}-1164 f_{4}+20 f_{5}-3102\right)+\left(15 b_{6}-588 b_{4}\right) b_{0}-4\left(9 f_{4}+5 f_{5}+288\right)\right)}{3\left(b_{0}^{2}-1\right)}, \\
\lambda_{7}=\frac{4}{105\left(b_{0}^{2}-1\right)}\left[b_{0}^{2}\left(680 \mathrm{~b}_{5}-151788 f_{4}+1740 f_{5}-412161\right)\right. \\
\left.-3\left(10 \mathrm{~b}_{5}+1404 f_{4}+580 f_{5}+46953\right)-390\left(196 b_{4}-5 b_{6}\right) b_{0}\right] .
\end{gathered}
$$

The first non-trivial coefficient in the expansion of the blackening factor appears at order $u^{5}$ due to the D2-brane asymptotics imposed and is undetermined. The expansion can be written as

$$
\mathrm{b}=1+\sum_{n=5}^{23} \mathrm{~b}_{n} u^{n}+\mathcal{O}\left(u^{24}\right),
$$


with the parameters

$$
\begin{gathered}
\mathrm{b}_{6}=\frac{20 \mathrm{~b}_{5}}{3}, \quad \mathrm{~b}_{7}=\frac{240 \mathrm{~b}_{5}}{7}, \quad \mathrm{~b}_{8}=160 \mathrm{~b}_{5}, \quad \mathrm{~b}_{9}=\frac{6400 \mathrm{~b}_{5}}{9}, \\
\mathrm{~b}_{10}=\frac{1}{20} \mathrm{~b}_{5}\left(4 f_{4}-20 f_{5}+60513\right), \\
\mathrm{b}_{11}=-\frac{2 \mathrm{~b}_{5}}{33\left(b_{0}^{2}-1\right)}\left[b_{0}^{2}\left(25 \mathrm{~b}_{5}-5916 f_{4}+220 f_{5}-223857\right)+\left(75 b_{6}-2940 b_{4}\right) b_{0}\right. \\
\left.\quad-84 f_{4}-220 f_{5}+202587\right] .
\end{gathered}
$$

Likewise, the leading order in the expansion of the warp factor is $u^{5}$ so that the D2brane asymptotics is maintained

$$
\mathbf{h}=\sum_{n=5}^{27} h_{n} u^{n}+\mathcal{O}\left(u^{28}\right)
$$

The first few coefficients are

$$
\begin{aligned}
& h_{5}=-\frac{16}{15}\left(b_{0}^{2}-1\right), \quad h_{6}=-\frac{64}{9}\left(2 b_{0}^{2}-1\right), \quad h_{7}=-\frac{64}{315}\left(581 b_{0}^{2}-201\right), \\
& h_{8}=-\frac{512}{45}\left(69 b_{0}^{2}-19\right), \quad h_{9}=-\frac{1024}{945}\left(4263 b_{0}^{2}-1003\right), \\
& h_{10}= \frac{4\left(7 b_{0}^{2}\left(252 f_{4}+180 f_{5}-1387925\right)+784 b_{4} b_{0}-5\left(84 f_{4}+252 f_{5}-401183\right)\right)}{1575}, \\
& h_{11}= \frac{32}{10395}\left[21 b_{0}^{2}\left(85 b_{5}-15904 f_{4}+1000 f_{5}-1896225\right)-21\left(8092 b_{4}-225 b_{6}\right) b_{0}\right. \\
&\left.-4\left(3213 f_{4}+3465 f_{5}-1794298\right)\right] .
\end{aligned}
$$

Finally, the fluxes are expanded as

$\mathcal{B}_{J}=\sum_{n=0}^{23} \mathcal{B}_{J, n} u^{n}+\mathcal{O}\left(u^{24}\right), \quad \mathcal{B}_{X}=\sum_{n=0}^{23} \mathcal{B}_{X, n} u^{n}+\mathcal{O}\left(u^{24}\right), \quad \mathcal{A}_{J}=\sum_{n=0}^{22} \mathcal{B}_{J, n} u^{n}+\mathcal{O}\left(u^{23}\right)$.

For convenience, we keep the undetermined parameters in the same function, namely $\mathcal{B}_{J}$. The parameter $b_{0}$ labelling the representative of the family is the leading order of both $\mathcal{B}_{J}$ and $\mathcal{B}_{X}$. The following coefficients in the expansion of $\mathcal{B}_{J}$ are

$$
\begin{aligned}
\mathcal{B}_{J, 1}=4 b_{0}, \quad \mathcal{B}_{J, 2}= & 8 b_{0}, \quad \mathcal{B}_{J, 3}=-16 b_{0}, \quad \mathcal{B}_{J, 5}=\frac{8}{5} b_{0}\left(8 f_{4}+45\right)+\frac{34 b_{4}}{5} \\
\mathcal{B}_{J, 7}=-\frac{4}{525\left(b_{0}^{2}-1\right)}[ & b_{0}^{3}\left(108 b_{5}+21108 f_{4}-244 f_{5}+246963\right) \\
& -b_{0}\left(178 b_{5}+4308 f_{4}-244 f_{5}+187407\right) \\
& \left.+42\left(139 b_{4}-20 b_{6}\right) b_{0}^{2}+126\left(19 b_{4}+5 b_{6}\right)\right]
\end{aligned}
$$


while for $\mathcal{B}_{X}$ we have

$$
\begin{gathered}
\mathcal{B}_{X, 1}=4 b_{0}, \quad \mathcal{B}_{X, 2}=12 b_{0}, \quad \mathcal{B}_{X, 3}=32 b_{0}, \quad \mathcal{B}_{X, 4}=-64 b_{0}-\frac{b_{4}}{2}, \\
\mathcal{B}_{X, 5}=\frac{1}{5}\left(-b_{0}\left(56 f_{4}+1755\right)-6 b_{4}\right), \quad \mathcal{B}_{X, 6}=-16 b_{0}\left(8 f_{4}+93\right)-42 b_{4}+b_{6} \\
\mathcal{B}_{X, 7}=\frac{2}{525\left(b_{0}^{2}-1\right)}[ \\
{\left[b_{0}^{3}\left(72 b_{5}-87918 f_{4}-46 f_{5}-511593\right)\right.} \\
+4 b_{0}\left(173 b_{5}+29118 f_{4}+46 f_{5}+303147\right) \\
\left.-21\left(7332 b_{4}-185 b_{6}\right) b_{0}^{2}+38724 b_{4}-945 b_{6}\right] .
\end{gathered}
$$

The remaining flux, $\mathcal{A}_{J}$, has coefficients

$$
\begin{aligned}
\mathcal{A}_{J, 0}= & \frac{b_{0}}{6}, \quad \mathcal{A}_{J, 1}=\frac{4 b_{0}}{3}, \quad \mathcal{A}_{J, 2}=8 b_{0}, \quad \mathcal{A}_{J, 3}=\frac{1}{6}\left(-32 b_{0}-b_{4}\right) \\
\mathcal{A}_{J, 4}= & b_{0}\left(-2 f_{4}-\frac{167}{12}\right)-\frac{2 b_{4}}{3}, \quad \mathcal{A}_{J, 5}=-\frac{16}{15}\left[b_{0}\left(8 f_{4}+45\right)+3 b_{4}\right] \\
\mathcal{A}_{J, 6}= & \frac{1}{225\left(b_{0}^{2}-1\right)}\left[b_{0}^{3}\left(4 b_{5}-3\left(5732 f_{4}+44 f_{5}+22325\right)\right)\right. \\
& \left.+3 b_{0}\left(12 b_{5}+2532 f_{4}+44 f_{5}+10981\right)-24\left(336 b_{4}-5 b_{6}\right) b_{0}^{2}+3360 b_{4}\right] \\
\mathcal{A}_{J, 7}=- & \frac{4}{17325\left(b_{0}^{2}-1\right)}\left[b_{0}^{3}\left(7594 b_{5}+1537164 f_{4}+39748 f_{5}+5893635\right)\right. \\
& -b_{0}\left(12004 b_{5}+478764 f_{4}+39748 f_{5}+2141607\right)+84\left(9204 b_{4}-145 b_{6}\right) b_{0}^{2} \\
& \left.-210\left(1212 b_{4}+5 b_{6}\right)\right] .
\end{aligned}
$$

\section{Expansions near the horizon}

In this appendix we consider the expansion around the horizon, eq. (3.7). Although we have solved the equations to sixth order, we only show the first term for the different functions. They read

$$
\begin{aligned}
f_{1}^{h}= & \frac{\lambda_{h}^{2}\left(9 h_{h}\left(f_{h}^{4}+g_{h}^{4}\right)+\left(-6 \alpha_{h}+\xi_{h}+\chi_{h}\right)^{2}\right)-2 g_{h}^{2}\left(9 h_{h} g_{h}^{2}\left(g_{h}^{2}-3 f_{h}^{2}\right)+\left(\xi_{h}-\chi_{h}\right)^{2}\right)}{18 h_{h} \mathrm{~b}_{h} f_{h}^{3} g_{h}^{4} u_{h}^{4}}, \\
g_{1}^{h}= & \frac{g_{h}^{2} \lambda_{h}^{2}\left(9 h_{h} f_{h}^{4}+2\left(6 \alpha_{h}+\xi_{h}\right)^{2}\right)+f_{h}^{4}\left(9 h_{h}\left(f_{h}^{4}+g_{h}^{4}\right)-\left(\xi_{h}-\chi_{h}\right)^{2}\right)}{9 h_{h} \mathrm{~b}_{h} f_{h}^{8} g_{h} u_{h}^{4}}, \\
\lambda_{1}^{h}= & \frac{\lambda_{h}}{9 h_{h} \mathrm{~b}_{h} f_{h}^{8} g_{h}^{4} u_{h}^{4}}\left[f_{h}^{4}\left(18 h_{h} g_{h}^{4} \lambda_{h}^{2}-2 g_{h}^{2}\left(\xi_{h}-\chi_{h}\right)^{2}+\lambda_{h}^{2}\left(-6 \alpha_{h}+\xi_{h}+\chi_{h}\right)^{2}\right)\right. \\
& \left.+9 h_{h} f_{h}^{8} \lambda_{h}^{2}+2 g_{h}^{4} \lambda_{h}^{2}\left(6 \alpha_{h}+\xi_{h}\right)^{2}\right], \\
h_{1}^{h}= & -\frac{\lambda_{h}^{2}}{81 h_{h} \mathrm{~b}_{h} f_{h}^{8} g_{h}^{4} u_{h}^{4}}\left[18 h_{h} f_{h}^{4}\left(9 h_{h} g_{h}^{4}+\left(-6 \alpha_{h}+\xi_{h}+\chi_{h}\right)^{2}\right)+81 h_{h}^{2} f_{h}^{8}\right. \\
& \left.+36 h_{h} g_{h}^{4}\left(6 \alpha_{h}+\xi_{h}\right)^{2}+\left(2 \chi_{h}\left(6 \alpha_{h}+\xi_{h}\right)-12 \alpha_{h} \xi_{h}+\xi_{h}^{2}-3\right)^{2}\right],
\end{aligned}
$$




$$
\begin{aligned}
& \mathrm{b}_{1}^{h}= \frac{9 h_{h} f_{h}^{4}\left(-\mathrm{b}_{h} g_{h}^{2} u_{h}^{3}-1\right)-54 h_{h} f_{h}^{2} g_{h}^{2}+9 h_{h} g_{h}^{4}+\left(\xi_{h}-\chi_{h}\right)^{2}}{9 h_{h} f_{h}^{4} g_{h}^{2} u_{h}^{4}} \\
& \mathcal{B}_{J, 1}^{h}= \frac{1}{9 h_{h} \mathrm{~b}_{h} f_{h}^{4} g_{h}^{4} u_{h}^{4}}\left[9 h_{h} f_{h}^{4}\left(2 g_{h}^{2}\left(\xi_{h}-\chi_{h}\right)+\lambda_{h}^{2}\left(-6 \alpha_{h}+\xi_{h}+\chi_{h}\right)\right)\right. \\
&+\lambda_{h}^{2}\left(18 h_{h} g_{h}^{4}\left(6 \alpha_{h}+\xi_{h}\right)+\left(6 \alpha_{h}-\xi_{h}-\chi_{h}\right)\left(12 \alpha_{h}\left(\xi_{h}-\chi_{h}\right)-\xi_{h}\left(\xi_{h}+2 \chi_{h}\right)\right)\right. \\
& \quad\left.\left.-3\left(\xi_{h}+\chi_{h}\right)\right)+18 \alpha_{h} \lambda_{h}^{2}\right] \\
& \mathcal{B}_{X, 1}^{h}=\frac{1}{9 h_{h} \mathrm{~b}_{h} f_{h}^{8} u_{h}^{4}}\left[18 h_{h} f_{h}^{4}\left(2 g_{h}^{2}\left(\chi_{h}-\xi_{h}\right)+\lambda_{h}^{2}\left(-6 \alpha_{h}+\xi_{h}+\chi_{h}\right)\right)\right. \\
&\left.\quad-2 \lambda_{h}^{2}\left(6 \alpha_{h}+\xi_{h}\right)\left(12 \alpha_{h}\left(\xi_{h}-\chi_{h}\right)-\xi_{h}\left(\xi_{h}+2 \chi_{h}\right)+3\right)\right] \\
& \mathcal{A}_{J, 1}^{h}=-\frac{1}{27 h_{h} \mathrm{~b}_{h} f_{h}^{4} g_{h}^{2} u_{h}^{4}}\left[9 h_{h} f_{h}^{4}\left(-6 \alpha_{h}+\xi_{h}+\chi_{h}\right)-18 h_{h} g_{h}^{4}\left(6 \alpha_{h}+\xi_{h}\right)\right. \\
&\left.\quad+\left(\xi_{h}-\chi_{h}\right)\left(-12 \alpha_{h}\left(\xi_{h}-\chi_{h}\right)+2 \xi_{h} \chi_{h}+\xi_{h}^{2}-3\right)\right]
\end{aligned}
$$

\section{Numerical output}

In this appendix we show some of the data obtained in our numerical computation. First of all, in figure 16 (left) we show the value of the functions $e^{\mathcal{F}}, e^{\mathcal{G}}$ and $e^{\Lambda}$ for some representative values of $b_{0}$, as a function of the position of the horizon normalized to the point where the regular solution ends. Recall that we already argued that there was a maximum $u_{N}<u_{s}$ above which we cannot place the horizon. This can be clearly seen in figure 16 (left), since the values at the horizon go to zero at some point $u_{N} / u_{s}<1$. Moreover, in these plots we see that as $b_{0}$ increases, the ratio between $u_{N}$ and $u_{s}$ decreases. For completeness, in figure 16 (right) we show the value of the fluxes at the horizon.

It is often the case that, when "heating up" a geometry, the value of the scalar fields at the horizon is similar to those in the zero-temperature solution at the same value of the radial coordinate (this statement is meaningful if the radial gauge is completely fixed, and in the same way, in both geometries). In our case this does not happen, as can be seen, for example, in the plot of $e^{\Lambda}$ : whilst for the supersymmetric solutions (both regular and irregular) the function $e^{\Lambda}$ is monotonically decreasing, its horizon values develop a minimum and a maximum, resulting in a manifest difference in the dilaton behaviour.

In figure 17 we show the value of the warp factor at the horizon and the value of $\mathrm{b}_{h}$, which is the derivative of the blackening factor at the horizon. Notice that both magnitudes diverge as $u_{h}$ approach $u_{N}$ : it is the contribution of both divergences (one in the denominator and the other in the numerator) that gives a finite temperature in the zero-entropy limit.

Open Access. This article is distributed under the terms of the Creative Commons Attribution License (CC-BY 4.0), which permits any use, distribution and reproduction in any medium, provided the original author(s) and source are credited. 

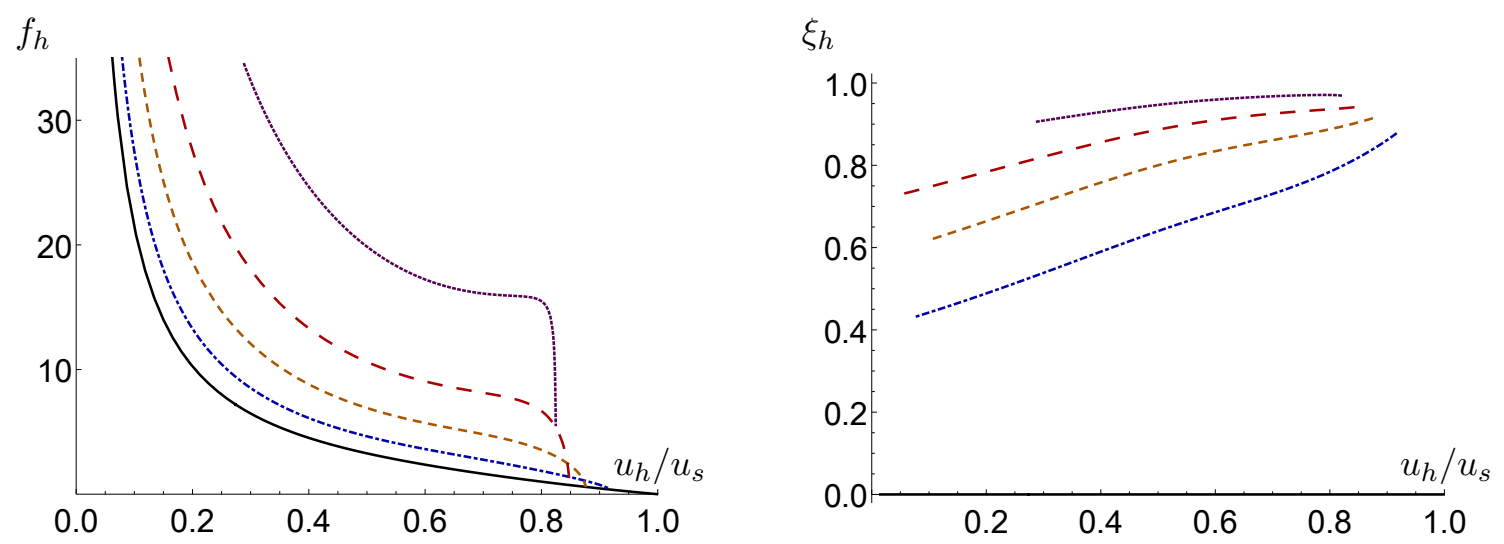

$g_{h}$

$\chi_{h}$
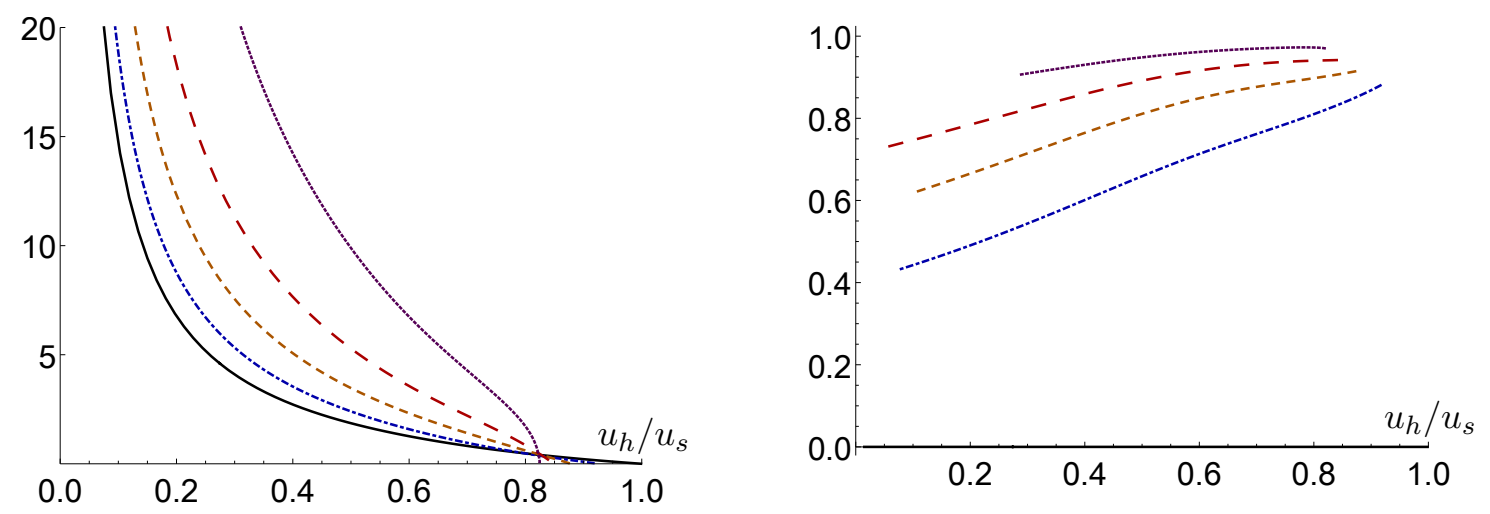

$\lambda_{h}$
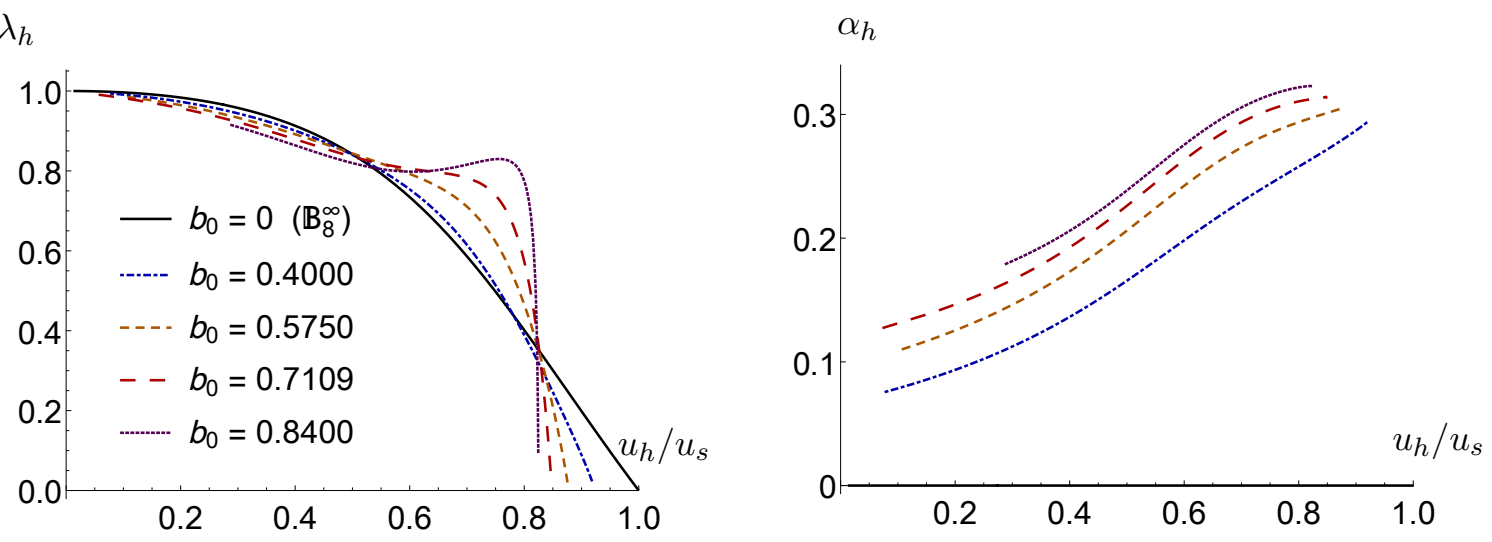

Figure 16. Value of the functions $e^{\mathcal{F}}, e^{\mathcal{G}}$ and $e^{\Lambda}$ at the horizon as a function of the position of the horizon normalized to $u_{s}$ for the metrics showed in the legend (left). Value of the fluxes at the horizon as a function of the position of the horizon normalized to $u_{s}$ for the metrics showed in the legend (right). 

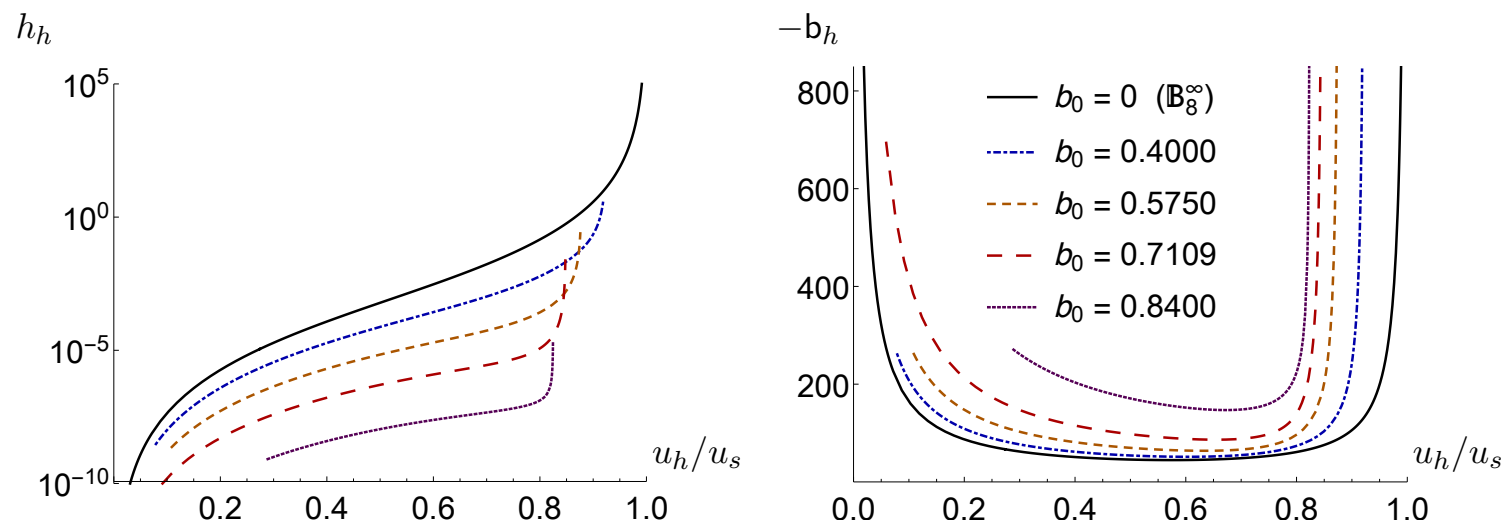

Figure 17. Value of the warp factor at the horizon (left) and value of the derivative of the blackening factor at the horizon (right) as a function of the position of the horizon normalized to $u_{s}$.

\section{References}

[1] J. Braun, H. Gies, L. Janssen and D. Roscher, Phase structure of many-flavor $Q E D_{3}$, Phys. Rev. D 90 (2014) 036002 [arXiv: 1404.1362] [InSPIRE].

[2] Z. Komargodski and N. Seiberg, A symmetry breaking scenario for $Q C D_{3}$, JHEP 01 (2018) 109 [arXiv: 1706. 08755] [INSPIRE].

[3] J. Gomis, Z. Komargodski and N. Seiberg, Phases Of Adjoint QCD ${ }_{3}$ And Dualities, SciPost Phys. 5 (2018) 007 [arXiv:1710.03258] [INSPIRE].

[4] C. Choi, M. Ro cek and A. Sharon, Dualities and Phases of $3 D N=1$ SQCD, JHEP 10 (2018) 105 [arXiv: 1808. 02184] [INSPIRE].

[5] E. Witten, Supersymmetric index of three-dimensional gauge theory, in M.A. Shifman ed. The many faces of the superworld, World Scientific (2000), pp. 156-184, hep-th/9903005 [INSPIRE].

[6] M.C. Diamantini, P. Sodano and C.A. Trugenberger, Oblique confinement and phase transitions in Chern-Simons gauge theories, Phys. Rev. Lett. 75 (1995) 3517 [cond-mat/9407073] [INSPIRE].

[7] P.H. Damgaard, U.M. Heller, A. Krasnitz and T. Madsen, A quark-antiquark condensate in three-dimensional QCD, Phys. Lett. B 440 (1998) 129 [hep-lat/9803012] [INSPIRE].

[8] N. Karthik and R. Narayanan, Bilinear condensate in three-dimensional large- $N_{c} Q C D$, Phys. Rev. D 94 (2016) 045020 [arXiv: 1607.03905] [INSPIRE].

[9] I.R. Klebanov and E. Witten, Superconformal field theory on three-branes at a Calabi-Yau singularity, Nucl. Phys. B 536 (1998) 199 [hep-th/9807080] [INSPIRE].

[10] A.F. Faedo, D. Mateos, D. Pravos and J.G. Subils, Mass Gap without Confinement, JHEP 06 (2017) 153 [arXiv: 1702.05988] [INSPIRE].

[11] M. Cvetič, G.W. Gibbons, H. Lü and C.N. Pope, New cohomogeneity one metrics with spin(7) holonomy, J. Geom. Phys. 49 (2004) 350 [math/0105119] [INSPIRE].

[12] M. Cvetič, G.W. Gibbons, H. Lü and C.N. Pope, New complete noncompact spin(7) manifolds, Nucl. Phys. B 620 (2002) 29 [hep-th/0103155] [INSPIRE]. 
[13] I.R. Klebanov and M.J. Strassler, Supergravity and a confining gauge theory: Duality cascades and $\chi$ SB resolution of naked singularities, JHEP 08 (2000) 052 [hep-th/0007191] [INSPIRE].

[14] E. Witten, Anti-de Sitter space, thermal phase transition and confinement in gauge theories, Adv. Theor. Math. Phys. 2 (1998) 505 [hep-th/9803131] [INSPIRE].

[15] A. Buchel, Klebanov-Strassler black hole, JHEP 01 (2019) 207 [arXiv:1809. 08484] [INSPIRE].

[16] O. Aharony, A. Buchel and P. Kerner, The black hole in the throat: Thermodynamics of strongly coupled cascading gauge theories, Phys. Rev. D 76 (2007) 086005 [arXiv:0706.1768] [INSPIRE].

[17] A. Loewy and Y. Oz, Branes in special holonomy backgrounds, Phys. Lett. B 537 (2002) 147 [hep-th/0203092] [INSPIRE].

[18] A. Hashimoto, S. Hirano and P. Ouyang, Branes and fluxes in special holonomy manifolds and cascading field theories, JHEP 06 (2011) 101 [arXiv:1004.0903] [INSPIRE].

[19] M. Cvetič, G.W. Gibbons, H. Lü and C.N. Pope, Supersymmetric nonsingular fractional D-2 branes and NS-NS 2 branes, Nucl. Phys. B 606 (2001) 18 [hep-th/0101096] [InSPIRE].

[20] C.P. Herzog, String tensions and three-dimensional confining gauge theories, Phys. Rev. D 66 (2002) 065009 [hep-th/0205064] [INSPIRE].

[21] H. Ooguri and C.-S. Park, Superconformal Chern-Simons Theories and the Squashed Seven Sphere, JHEP 11 (2008) 082 [arXiv:0808.0500] [INSPIRE].

[22] M. Attems, Y. Bea, J. Casalderrey-Solana, D. Mateos, M. Triana and M. Zilhão, Holographic Collisions across a Phase Transition, Phys. Rev. Lett. 121 (2018) 261601 [arXiv: 1807.05175] [INSPIRE].

[23] M. Attems, Y. Bea, J. Casalderrey-Solana, D. Mateos, M. Triana and M. Zilhao, Phase Transitions, Inhomogeneous Horizons and Second-Order Hydrodynamics, JHEP 06 (2017) 129 [arXiv: 1703.02948] [INSPIRE].

[24] M. Attems, Y. Bea, J. Casalderrey-Solana, D. Mateos and M. Zilhão, Dynamics of Phase Separation from Holography, JHEP 01 (2020) 106 [arXiv: 1905.12544] [INSPIRE].

[25] R.A. Janik, J. Jankowski and H. Soltanpanahi, Real-Time dynamics and phase separation in a holographic first order phase transition, Phys. Rev. Lett. 119 (2017) 261601 [arXiv: 1704.05387] [INSPIRE].

[26] L. Bellantuono, R.A. Janik, J. Jankowski and H. Soltanpanahi, Dynamics near a first order phase transition, JHEP 10 (2019) 146 [arXiv:1906.00061] [INSPIRE].

[27] Y. Bea et al., Crossing a large- $N_{c}$ phase transition at finite volume, to appear.

[28] S.S. Gubser, Curvature singularities: The Good, the bad and the naked, Adv. Theor. Math. Phys. 4 (2000) 679 [hep-th/0002160] [INSPIRE].

[29] D. Elander, A.F. Faedo, D. Mateos, D. Pravos and J.G. Subils, Mass spectrum of gapped, non-confining theories with multi-scale dynamics, JHEP 05 (2019) 175 [arXiv:1810.04656] [INSPIRE].

[30] P. Basu, F. Nogueira, M. Rozali, J.B. Stang and M. Van Raamsdonk, Towards A Holographic Model of Color Superconductivity, New J. Phys. 13 (2011) 055001 [arXiv:1101.4042] [INSPIRE]. 
[31] Ó.J.C. Dias, G.S. Hartnett, B.E. Niehoff and J.E. Santos, Mass-deformed M2 branes in Stenzel space, JHEP 11 (2017) 105 [arXiv: 1704. 02323] [INSPIRE].

[32] G.C. Giecold, Finite-Temperature Fractional D2-branes and the Deconfinement Transition in 2+1 Dimensions, JHEP 03 (2010) 109 [arXiv: 0912.1558] [INSPIRE].

[33] I. Bena, Ó.J.C. Dias, G.S. Hartnett, B.E. Niehoff and J.E. Santos, Holographic dual of hot Polchinski-Strassler quark-gluon plasma, JHEP 09 (2019) 033 [arXiv:1805.06463] [INSPIRE].

[34] I. Bena, A. Buchel and O.J.C. Dias, Horizons cannot save the Landscape, Phys. Rev. D 87 (2013) 063012 [arXiv: 1212.5162] [INSPIRE].

[35] I. Bena, J. Blaback, U.H. Danielsson and T. Van Riet, Antibranes cannot become black, Phys. Rev. D 87 (2013) 104023 [arXiv:1301.7071] [InSPIRE].

[36] D. Cohen-Maldonado, J. Diaz, T. van Riet and B. Vercnocke, Observations on fluxes near anti-branes, JHEP 01 (2016) 126 [arXiv:1507.01022] [INSPIRE].

[37] D. Cohen-Maldonado, J. Diaz and F.F. Gautason, Polarised antibranes from Smarr relations, JHEP 05 (2016) 175 [arXiv: 1603.05678] [INSPIRE].

[38] G.S. Hartnett, Localised Anti-Branes in Flux Backgrounds, JHEP 06 (2015) 007 [arXiv: 1501.06568] [INSPIRE].

[39] J. Armas, N. Nguyen, V. Niarchos, N.A. Obers and T. Van Riet, Meta-stable non-extremal anti-branes, Phys. Rev. Lett. 122 (2019) 181601 [arXiv:1812.01067] [INSPIRE].

[40] J. Armas, N. Nguyen, V. Niarchos and N.A. Obers, Thermal transitions of metastable M-branes, JHEP 08 (2019) 128 [arXiv:1904.13283] [INSPIRE].

[41] S. Kachru, R. Kallosh, A.D. Linde and S.P. Trivedi, de Sitter vacua in string theory, Phys. Rev. D 68 (2003) 046005 [hep-th/0301240] [INSPIRE].

[42] R. Emparan, C.V. Johnson and R.C. Myers, Surface terms as counterterms in the AdS/CFT correspondence, Phys. Rev. D 60 (1999) 104001 [hep-th/9903238] [INSPIRE]. 